\title{
Coxeter Groups
}

\section{and three Related Topics}

\author{
Arjeh M. Cohen \\ Centre for Mathematics and Computer Science \\ Kruislaan 413, 1098 SJ Amsterdam \\ and \\ Math. Inst., Rijksuniversiteit Utrecht \\ PO Box 80010,3508 TA Utrecht \\ The Netherlands \\ Notes of lectures given at \\ the ASI "Generators and Relations in Groups and Geometries"
}

\begin{abstract}
The theory of Coxeter groups is surveyed. The three related topics referred to in the title are Tits geometries, computational Lie group theory, and Hecke algebras.
\end{abstract}

\section{Outline}

Coxeter groups satisfy a number of amazing properties and play an important rôle in various branches of mathematics such as the theories of lattices, Lie groups and geometries, finite groups and graphs. We shall first develop the basic theory of Coxeter groups, along the lines of [Bourb 1968]. In particular, we shall discuss their faithful reflection representations, root systems, and the determination of all finite Coxeter groups.

Our next topic will be geometry. The defining presentation of a Coxeter group $W$ gives rise to a Cayley graph, which is usually called apartment. Quotients of this graph (which are proper graphs in the sense that the edges have no labels) represent the 'natural geometries' attached to $W$. Some of the simplest of these geometries are the polygons, the simplices and the hypercubes; they are important because of their ubiquity in the geometries related to groups of Lie type, the so-called buildings. The presentation of this part will be largely based on [BCN 1989] and [BuCo 1990].

The second related topic concerns computational aspects. From an algorithmic point of view, the Coxeter groups are among the easiest examples of groups presented by generators and relations to deal with; still they represent considerable problems. For instance, although the word problem is solvable, and despite some recent partially successful attempts, no 'efficient' set of rewrite rules has been found in the general case. These algorithms are of use in studying Lie group representations; we shall indicate how.

We finish by briefly touching upon a third related topic: Hecke algebras. They link 
the Coxeter groups with representation theory of algebraic groups and geometry related algebras. We provide the elementary definitions and give some references to the literature.

\section{Basic definitions}

A Coxeter matrix of rank $n$ is an $n \times n$ matrix $M=\left(m_{i, j}\right)_{1 \leq i, j \leq n}$ with $m_{i, i}=1$ and $m_{i, j}=m_{j, i}>1$ (possibly $\infty$ ) for all $i, j \in\{1, \ldots, n\}$ with $i \neq j$. The Coxeter group associated with the Coxeter matrix $M$ is the group generated by elements $\rho_{i}(i=1, \ldots, n)$ subject to the relations

$$
\left(\rho_{i} \rho_{j}\right)^{m_{i, j}}=1
$$

It is denoted by $W(M)$ or just $W$. Furthermore, we set $I=\{1, \ldots, n\}$ and $R=\left\{\rho_{i} \mid i \in I\right\}$ (both sets will be assumed finite throughout). The pair $(W, R)$ is called the Coxeter system of type $M$. The number $n$ is called the rank of the system (or group).

It is common practice to provide a pictorial presentation of $M$ by means of the labeled graph $(I, M)$ with vertex set $I$; the pair $\{i, j\}$ is an edge whenever $m_{i, j}>2$; this edge is labeled $m_{i, j}$. If $m_{i, j}=3$, the label is often omitted.

Three very important tools in the study of a Coxeter group are the monoid of words in the generators, the length function and the chamber system. They can be defined in great generality:

Let $I$ be an index set and $W$ any group generated by a set $R=\left\{\rho_{i} \mid i \in I\right\}$ of involutions. The free monoid on the alphabet $I$ with unit (usually denoted by $\epsilon$ ) is denoted by $I^{*}$ and $\rho: I^{*} \rightarrow W(R)$ stands for the monoid morphism determined by $\rho(i)=\rho_{i}(i \in I)$.

There is a natural notion of length for an element of $I^{*}$; the length of the empty element is 0 , the length of an element of the alphabet $I$ equals 1 , and so on. A typical element of $I^{*}$ will be written as $\mathbf{i}$ and its length as $l(\mathbf{i})$. Thus, if $l(\mathbf{i})=q$, there are $i_{j} \in I(1 \leq j \leq q)$ such that $\mathbf{i}=i_{1} \cdots i_{q}$. The length of an element $w \in W$, denoted by $l(w)$, or $l_{R}(w)$ if more precision is required, is $\min \{l(\mathbf{i}) \mid \rho(\mathbf{i})=w\}$. For each element $\mathbf{i}=i_{1} \cdots i_{q} \in I^{*}$ with $\rho(\mathbf{i})=w$, we call the product $\rho\left(i_{1}\right) \cdots \rho\left(i_{q}\right)$ an expression of $w$. If $q=l(w)$, the expression is called reduced. For arbitrary groups $W$, the restriction of $\rho$ to $I$ need not be injective; but for Coxeter groups $W$, it is (cf. Corollary 4.3).

The chamber system associated with $(W, R)$, denoted by $\mathcal{C}(W, R)$ or just $\mathcal{C}$, is the labeled graph whose vertex set is $W$ and in which the edges labeled $r$ (for $r \in R$ ) are all $\{w, w r\}$ for $w \in W$. Its (label preserving) automorphism group contains $W$ via left multiplication. Observe that the graph-theoretic distance between the 'chambers' $w, w^{\prime} \in W$ of $\mathcal{C}$ equals $l\left(w^{-1} w^{\prime}\right)$. The above definition of chamber system comes close to what is known as a Cayley graph, but the present choice of name is in accordance with the more general notion of chamber system to be treated in $\S 7$.

\section{Examples}

For the time being, let $(W, R)$ be a Coxeter system of type $M$ on $n=|R|$ generators.

3.1 First Examples (i) If $n=1$ then $W=\{1\} \cup R \cong \mathbb{Z} /(2)$, the group of order 2 .

(ii) If $n=2$, then $W \cong\left\langle r, s \mid r^{2}=s^{2}=(r s)^{m}=1\right\rangle=\operatorname{Dih}_{2 m}$, the dihedral group of order $2 m$, where $m=m_{1,2}$. In this case, we usually write $M=I_{2}^{m}$. 
(iii) The symmetric group $\operatorname{Sym}_{n+1}$ on $n+1$ letters is the Coxeter group $W\left(A_{n}\right)$ where

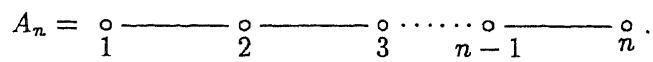

The evident morphism $W \rightarrow \operatorname{Sym}_{n+1}$ sending $\rho_{i}$ to $(i, i+1)$ for each $i \in I$ is an isomorphism. This will follow from later developments, but can also be proved by establishing by induction on $n$ that $\left\langle\rho_{1}, \ldots, \rho_{n-1}\right\rangle \cong W\left(A_{n-1}\right) \cong \operatorname{Sym}_{n}$ and that each element of $W\left(A_{n}\right) \backslash W\left(A_{n-1}\right)$ can be written as $w \rho_{n} w^{\prime}$ for certain $w, w^{\prime} \in W\left(A_{n-1}\right)$.

(iv) If $I=I_{1} \cup I_{2}$ is a partitioning of $(I, M)$ into disjoint graphs (here disjoint means: if $m_{i, j}=2$ whenever $i \in I_{1}$ and $\left.j \in I_{2}\right)$, then $W(M)=W\left(M_{1}\right) \times W\left(M_{2}\right)$, where $M_{k}$ is the restriction of $M$ to $I_{k} \times I_{k}(k=1,2)$. This explains why, in addressing many questions concerning Coxeter groups, we can restrict to the case where $M$ is connected.

(v) If $\mu:(I, M) \rightarrow(\bar{I}, \bar{M})=\left(\bar{m}_{i, j}\right)_{i, j \in \bar{I}}$ is a surjective morphism of Coxeter diagrams such that $\bar{m}_{\mu i, \mu j}$ divides $m_{i, j}$ for all $i, j \in I$, then there is a surjective morphism, also denoted by $\mu$, from $W$ to $W(\bar{M})$ such that $\mu \rho_{i}=\rho_{\mu i}$. The particular case where $\bar{M}$ has only one vertex leads to a surjective morphism $W \rightarrow \mathbb{Z} /(2)$. This map is known as the sign character; its kernel consists of all elements of even length.

The origin of Coxeter's interest in the groups bearing his name lies in the study of reflections in $n$-dimensional Euclidean space $A(V)$ corresponding to the real vector space $V$. A reflection in $A(V)$ is an affine transformation of the form $x \mapsto x-(x-b, a) a$ for $a, b \in V$ with $(a, a)=2$. Here, $(\cdot, \cdot)$ stands for the Euclidean inner product. The hyperplane $\{x \in V \mid(x, a)=(b, a)\}$ is the so-called reflection hyperplane of the reflection. If $b=0$, the reflection is a linear transformation and the vector $a$ spans its -1-eigenspace.

If a group $G$ acts on a set $E$, then a nonempty subset $P$ of $E$ is called a prefundamental domain for $G$ if $P \cap g P=\emptyset$ for all $g \in G, g \neq 1$. Thus, the existence of a prefundamental domain for $G$ implies that the action of $G$ on $E$ is faithful. Observe that a prefundamental domain need not quite be what is classically called a fundamental domain as it is not required that the domain be connected or contain a member of each $G$-orbit in $E$.

We are now ready to formulate a generalization of Coxeter's basic observation [Cox 1934] that Coxeter systems provide presentations for certain groups generated by reflections.

3.2 Theorem (cf. [Bourb 1968]) Let $\left\{H_{i}\right\}_{i \in I}$ be a family of affine hyperplanes of the real affine space $E$. For each $i \in I$, let $A_{i}$ denote one of the two open half-spaces determined by $H_{i}$. Assume that $A=\bigcap_{i \in I} A_{i} \neq \emptyset$. Furthermore, for each $i \in I$, let $\sigma_{i}$ be an affine reflection whose set of fixed points in $E$ is $H_{i}$. Assume that for $i \neq j$ in $I$, the intersection $A_{i j}=A_{i} \cap A_{j}$ is a prefundamental domain for the subgroup $G_{i j}$ of $A G L(V)$ generated by $\sigma_{i}$ and $\sigma_{j}$. Then

(i) $A$ is a prefundamental domain for the subgroup $G$ of $A G L(V)$ generated by the $\sigma_{i}$, $i \in I$

(ii) $\left(G,\left\{\sigma_{i} \mid i \in I\right\}\right)$ is a Coxeter system of type $M=\left(m_{i j}\right)_{i, j \in I}$, where $m_{i j}$ is the order of $\sigma_{i} \sigma_{j}$

(iii) for all $i \in I$ and $w \in W$, either $w A \subseteq A_{i}$ and $l\left(\rho_{i} w\right)=l(w)+1$, or $w A \subseteq \rho_{i} A_{i}$ and $l\left(\rho_{i} w\right)=l(w)-1$.

See [Vinb 1971] for more information on the structure of $\bigcup_{w \in W} w A$. Below we are primarily interested in linear groups generated reflections in real vector spaces. For characterizations of finite examples over fields of positive characteristic, see [Wag 1980/1] and [ZaSe 1981]. 
3.3 Further Examples (vi) Let $\Gamma$ be the cube in Euclidean space $\mathbb{R}^{3}$ whose vertices are the points all of whose coordinates are \pm 1 . Consider the incident triple consisting of the vertex $v_{1}=(1,1,1)$, the edge $v_{2}=\{(1,1,1),(1,1,-1)\}$, and the face $v_{3}=v_{2} U$ $\{(1,-1,1),(1,-1,-1)\}$. Denote by $\rho_{i}$ the reflection fixing $v_{i+1}$ and $v_{i+2}$ (indices $\bmod 3$ ). They are given by the matrices

$$
\left(\begin{array}{ccc}
1 & 0 & 0 \\
0 & 1 & 0 \\
0 & 0 & -1
\end{array}\right),\left(\begin{array}{lll}
1 & 0 & 0 \\
0 & 0 & 1 \\
0 & 1 & 0
\end{array}\right),\left(\begin{array}{lll}
0 & 1 & 0 \\
1 & 0 & 0 \\
0 & 0 & 1
\end{array}\right), \text { respectively. }
$$

The reflections $\rho_{1}, \rho_{2}, \rho_{3}$ leave $\Gamma$ invariant and generate the group $G$ of 48 isometries of $\Gamma$. This group is isomorphic to the Coxeter group $W\left(B_{3}\right)$, where, for arbitrary $n \geq 2$,

$$
B_{n}=\stackrel{\circ}{\circ}: \cdots \cdots \circ \frac{\cdots}{2} \div \frac{4}{n}-\frac{4}{n} .
$$

Here $A$ is a cone whose 'apex' is the origin and whose radii run through a small triangle in the face $v_{3}$.

(vii) Each of the convex regular polytopes of Euclidean space obtained in Theorem 3.2 gives rise to a group of isometries which is a Coxeter group. The $n$-simplex (with $n+1$ vertices) in $\mathbb{I R}^{n}$ gives $W\left(A_{n}\right)$, the hypercube in $\mathbb{I R}^{n}$ gives $W\left(B_{n}\right)$. As a result, besides $W\left(A_{n}\right)$ (of order $(n+1)$ !) and $W\left(B_{n}\right)$ (of order $2^{n} n$ !) the following Coxeter groups $W(M)$ can be shown to be finite; their orders can be computed by a count of images of $A$ in the same way as in (vi).

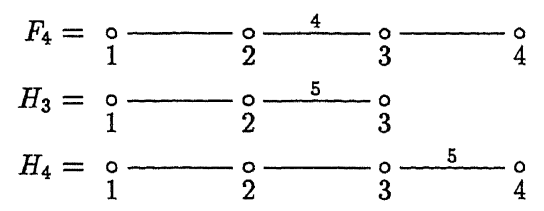

(viii) Consider the regular tiling of $\mathbb{I R}^{2}$ by triangles. Reflections in the hyperplanes bounding a triangle give a Coxeter group of type

$$
\tilde{G}_{2}=\stackrel{\circ}{\circ} \stackrel{6}{\circ} \stackrel{0}{2} \text {. }
$$

Higher dimensional analogues exist with diagrams

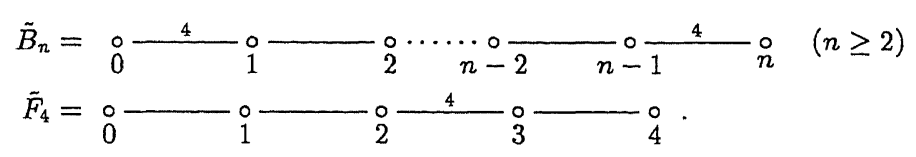

\section{Characterizations}

Obviously, any group generated by involutions is a quotient of a Coxeter group. Thus, in a sense, Coxeter groups are universal among groups generated by involutions (with specified 
orders for their products). Here are some other characterizations of Coxeter groups. For any set $X$, we write $\mathcal{P}(X)$ to denote its power set. If $\mathbf{i}=i_{1} \cdots i_{q} \in I^{*}$, then a substring of $\boldsymbol{i}$ is any word of the form $i_{\chi(1)} \cdots i_{\chi(t)}$ with $1 \leq \chi(1)<\ldots<\chi(t) \leq q$ for some $t \leq q$. The particular substring of length $q-1$ with $\chi(j)=j$ for precisely those $j$ for which $j<k$ is denoted by $i_{1} \cdots \widehat{i_{k}} \cdots i_{q}$. For any Coxeter system $(W, R)$, set $T=\bigcup_{w \in W} w R w^{-1}$ and write

$$
\mathbb{Z} /(2) T=\bigoplus_{t \in T} \mathbb{Z} /(2) t
$$

to denote the module with $W$-action given by $w \circ t=w t w^{-1}$ for $w \in W, t \in T$.

4.1 Theorem (Main Characterizations of Coxeter Groups) Let $W$ be a group generated by a set $R$ of involutions. Each of the following conditions on $W$ and $R$ is equivalent to $(W, R)$ being a Coxeter system.

(i) (Reflection Representation) There is a real linear representation $\sigma: W \rightarrow G L(V)$ such that, for $r \in R$, the transformation $\sigma(r)$ is a reflection with hyperplane $H_{r}$ and there is a choice of open half-space $A_{\boldsymbol{r}}$ with boundary $H_{r}$ such that

- $\bigcap_{r \in R} A_{r} \neq \emptyset$;

- $A_{r} \cap A_{s}$ is a prefundamental domain for $\langle r, s\rangle$ whenever $r, s \in R$.

(ii) (Root System) There exists a linear representation of $W$ on a real vector space $V$, a $W$-invariant set $\Phi$ of non-zero vectors in $V$, an embedding $r \mapsto e_{r}$ of $R$ into $\Phi$, and an ordering $\leq$ (partial) on $V$ (compatible with the real vector space structure of $V$ ) such that

- if $\alpha \in \Phi$ then $-\alpha \in \Phi$ and either $\alpha>0$ or $\alpha<0$;

$-e_{r}>0$ and $r e_{r}<0$ for all $r \in R$;

- if $\alpha \in \Phi \backslash\left\{e_{r}\right\}$ and $\alpha>0$, then $r \alpha>0$;

- if $w e_{r}=e_{r^{\prime}}$ then $r^{\prime}=w r w^{-1}$ for each $r^{\prime}, r \in R$ and $w \in W$.

(iii) (Cocycle Condition) There exists a map $\nu: W \rightarrow \mathbf{Z} /(2) T$ such that

$-\nu(r)=r$ for each $r \in R$;

$-\nu(v w)=\nu(v)+v \circ \nu(w)$ for all $v, w \in W$.

(iv) (Strong Exchange Condition) If $w=r_{1} \cdots r_{q} \in W$ (with $r_{i} \in R$ ) and $t \in T$ satisfy $l(t w) \leq l(w)$, then $t w=r_{1} \cdots \widehat{r}_{i} \cdots r_{q}$ for some $i \in\{1, \ldots, q\}$.

(v) (Bruhat Condition) There is a map Bruh: $W \rightarrow \mathcal{P}(W)$ such that

- if $w=r_{1} \cdots r_{q}$ is a reduced expression then Bruh( $\left.w\right)$ consists of all $r_{i_{1}} \cdots r_{i_{t}}$ for any substring $i_{1} \cdots i_{t}$ of $1 \cdots q$;

- for any $t \in T$ and $w \in W$ we have $w \in t \operatorname{Bruh}(w) \cup \operatorname{Bruh}(t w)$.

(vi) (Hyperplane Condition) There is a map $r \mapsto C_{r}$ from $R$ to $\mathcal{P}(W)$ such that

$-1 \in C_{r}$ for each $r \in R$;

- $C_{r} \cap r C_{r}=\emptyset$ for each $r \in R$;

- If $w \in W$ and $r, s \in R$ satisfy $w \in C_{r} \backslash C_{r} s$ then $r^{w}=s$.

(vii) (Exchange Condition) If $r_{1} \cdots r_{q} \in W$ (with $r_{i} \in R$ ) is a reduced expression for $w \in W$ and $r \in R$ satisfies $l(r w) \leq l(w)$, then $r w=r_{1} \cdots \widehat{r}_{i} \cdots r_{q}$ for some $i \in\{1, \ldots, q\}$.

In these cases, the type $M=\left(m_{r, s}\right)$ of $(W, R)$ is determined by the fact that $m_{r, s}$ equals the order of $r s$ for $r, s \in R$, and $C_{r}=\{w \in W \mid l(r w)>l(w)\}$.

All but (i) and (iii) of the above equivalent conditions are dealt with in [Deo 1986]; statement (iii) stems from [Dyer 1990]. We start here with the proof that a Coxeter system satisfies (i). Thus, we reverse the setting of the previous section in the sense that we produce 
a reflection group from a given Coxeter group. The outcome will be a linear reflection group rather than an affine one.

Consider a Coxeter system $(W, R)$ of type $M=\left(m_{i j}\right)_{i, j \in I}$. Let $V$ be a real vector space with basis $\left(e_{i}\right)_{i \in I}$ of vectors indexed by $I$. Denote by $B_{M}$, or just $B$ if $M$ is clear from the context, the symmetric bilinear form on $V$ defined by

$$
B\left(e_{i}, e_{j}\right)=-2 \cos \left(\pi / m_{i j}\right) \quad(i, j \in I)
$$

with the understanding that $B\left(e_{i}, e_{j}\right)=-2$ if $m_{i j}=\infty$. We call $B_{M}$, the symmetric bilinear form associated with $M$ and write $x \perp y$ to denote $B(x, y)=0$ for $x, y \in V$. For each $i \in I$, consider the linear transformation $\sigma_{i}$ of $V$ defined by

$$
\sigma_{i}(x)=x-B\left(x, e_{i}\right) e_{i} \quad(x \in V)
$$

This defines a reflection of $G L(V)$ in the hyperplane $e_{i}^{\perp}$ and with root (i.e., eigenvector with non-trivial eigenvalue) $e_{i}$.

The following general approach is due to Tits (cf. [Tits 1969], [Bourb 1968]).

4.2 Proposition (Reflection Representation) Let $B$ be the symmetric bilinear form as sociated with the Coxeter matrix $M$, and let $\rho_{i}(i \in I)$ be as in (1). Then the mapping $\gamma: R \rightarrow\left\{\sigma_{i} \mid i \in I\right\}$ given by $\gamma\left(\rho_{i}\right)=\sigma_{i}$ extends to an orthogonal representation $\gamma: W \rightarrow O\left(V, B_{M}\right)$ (that is, a linear representation on $V$ preserving $B_{M}$ ).

Proof Any vector $x \in V$ decomposes as $x=x_{i}+x^{\prime}{ }_{i}$, where $x_{i} \in \mathbb{R} e_{i}$ and $x^{\prime}{ }_{i} \in e_{i}^{\perp}$. Then $\sigma_{i}(x)=x_{i}^{\prime}-x_{i}$, so $B\left(\sigma_{i}(x), \sigma_{i}(y)\right)=B\left(x_{i}^{\prime}-x_{i}, y_{i}^{\prime}-y_{i}\right)=B\left(x_{i}^{\prime}, y_{i}^{\prime}\right)+B\left(x_{i}, y_{i}\right)=$ $B\left(x_{i}^{\prime}+x_{i}, y_{i}^{\prime}+y_{i}\right)=B(x, y)$.

Suppose $m_{i j}=\infty$. Then $B\left(e_{i}, e_{j}\right)=-2$ and $e_{i}+e_{j} \in e_{i}^{\frac{1}{1}} \cap e_{j}^{\frac{1}{j}}$, whence $e_{i}+e_{j}$ is fixed by $\sigma_{i}$ and $\sigma_{j}$. As $\sigma_{i} \sigma_{j}\left(e_{i}\right)=e_{i}+2\left(e_{i}+e_{j}\right)$, this yields $\left(\sigma_{i} \sigma_{j}\right)^{n}\left(e_{i}\right)=e_{i}+2 n\left(e_{i}+e_{j}\right)$ for each $n \in \mathbb{I N}$, so $\sigma_{i} \sigma_{j}$ has infinite order.

Suppose $m_{i j}<\infty$. Then, for $x=x_{i} e_{i}+x_{j} e_{j}$,

$$
\begin{aligned}
B(x, x) & =x_{i}^{2}-2 x_{i} x_{j} \cos \left(\pi / m_{i j}\right)+x_{j}^{2}= \\
& =\left(x_{i}-x_{j} \cos \left(\pi / m_{i j}\right)\right)^{2}+x_{j}^{2} \sin ^{2}\left(\pi / m_{i j}\right) .
\end{aligned}
$$

Thus, the restriction of $B$ to $\mathbb{R} e_{i}+\mathbb{R} e_{j}$ is positive definite, so the restrictions of $\sigma_{i}$ and $\sigma_{j}$ can be interpreted as reflections in a Euclidean plane. Since $B\left(e_{i}, e_{j}\right)=-2 \cos \left(\pi / m_{i j}\right)=$ $2 \cos \left(\pi-\pi / m_{i j}\right)$, the angle between $e_{i}$ and $e_{j}$ is $\pi-\pi / m_{i j}$. Thus the restriction of $\sigma_{i} \sigma_{j}$ to the plane $\mathbb{R} e_{i}+\mathbb{R} e_{j}$ is a rotation of angle $2 \pi / m_{i j}$, and so has order $m_{i j}$. Moreover $V=\left(\mathbb{R} e_{i}+\mathbb{R} e_{j}\right)+\left(e_{i}^{\perp} \cap e_{j}^{\perp}\right)$ and $\sigma_{i}$ and $\sigma_{j}$ act trivially on the second factor. Therefore, the order of $\sigma_{i} \sigma_{j}$ also equals $m_{i j}$.

QED

4.3 Corollary If $(W, R)$ is a Coxeter system of type $M$, then

(i) the mapping $i \mapsto \rho_{i}(i \in I)$ is a bijection from $I$ onto $R$;

(ii) the restriction of $\gamma$ to the subgroup $\left\langle\rho_{i}, \rho_{j}\right\rangle$ of $W$ is faithful for every $i, j \in I$.

We will employ Theorem 3.2 to derive that $\gamma$ itself is faithful. 
4.4 Examples (i) For $M=B_{3}$, there is an equivalence between the representations defined by the proposition above and Example 3.3(vi) such that $\sigma_{1}, \sigma_{2}, \sigma_{3}$ correspond to the reflections $\rho_{1}, \rho_{2}, \rho_{3}$ defined there. The bilinear form $B$ of the proposition corresponds to the standard inner product left invariant by the $\sigma_{i}$.

(ii) The infinite dihedral group. Let $I=\{1,2\}$ and $m_{12}=\infty$. On the basis $e_{1}, e_{2}$, the bilinear form $B$ of the proposition is

$$
B(x, y)=2 x_{1} y_{1}+2 x_{2} y_{2}-2 x_{1} y_{2}-2 x_{2} y_{1} \quad\left(x=x_{1} e_{1}+x_{2} e_{2}, y=y_{1} e_{1}+y_{2} e_{2}\right),
$$

so that $e_{1}+e_{2} \in e_{1}^{\perp} \cap e_{2}^{\perp}$. Hence $\sigma_{1}$ and $\sigma_{2}$ fix all points of the line $x_{1}-x_{2}=0$. We would like to apply Theorem 3.2 to derive that the representation $\gamma$ is faithful, but there is no convenient choice for $A$ as in Theorem 3.2. Therefore, we switch to the contragredient representation.

Consider the dual vector space $V^{*}$ and the contragredient representation $\gamma^{*}$ determined by $\langle f, v\rangle=\left\langle\gamma^{*}(g) f, \gamma(g) v\right\rangle$ for all $v \in V, f \in V^{*}$. For $v \in V$, set $A_{v}=\left\{x \in V^{*} \mid\langle x, v\rangle>0\right\}$. This is a half-open space in $V^{*}$. Now we set $A_{i}=A_{e_{i}}$ and $A=\bigcap_{i \in I} A_{i}$. Then $A \neq \emptyset$ (as $\sum_{i} e_{i}^{*} \in A$ where $\left(e_{i}^{*}\right)_{i}$ is the dual basis of $\left.\left(e_{i}\right)_{i}\right)$ and for $g \in W, v \in V$ we have $A_{\gamma(g) v}=\gamma^{*}(g) A_{v}$, since $\langle x, v\rangle>0$, is equivalent to $\left\langle\gamma^{*}(g) x, \gamma(g) v\right\rangle>0$. In particular, $\gamma^{*}\left(\rho_{i}\right) A=A_{\gamma\left(\rho_{i}\right) e_{i}}=A_{-e_{i}}=-A_{i}$, whence $A_{i} \cap \gamma^{*}\left(\rho_{i}\right) A_{i}=\emptyset$ for all $i \in I$.

4.5 Theorem In $V^{*}$, the half-spaces $A_{i}$ and the reflections $\gamma^{*}\left(\rho_{i}\right)$ satisfy the assumptions of Theorem 3.2. In particular, $\gamma^{*}$ and $\gamma$ are faithful.

Proof The second statement follows from $\operatorname{ker} \gamma=\operatorname{ker} \gamma^{*}$ and from Theorem 3.2. Next, $A=\bigcap_{i \in I} A_{i} \neq \emptyset$ since a linear form $f$ taking the value $I$ on each $e_{i}$ is definitely in $A$.

Finally, let $i \neq j$ in $I$ and consider $w \in W_{i j}$ with $A_{i j} \cap \gamma^{*}(w) A_{i j} \neq \emptyset$. We must show that $w=1$. To this end, consider the subspace $U=\mathbb{R} e_{i}+\mathbb{R} e_{j}$ in $V$. There is a canonical homomorphism $\pi$ from $V^{*}$ onto $U^{*}$ (namely restrictions of linear forms on $V$ to $U$ ). Since $\rho_{i}$ leaves invariant every plane containing $e_{i}$, in particular $U$ (and similarly for $\rho_{j}$ instead of $\left.\rho_{i}\right)$, the group $W_{i, j}=\left\langle\rho_{i}, \rho_{j}\right\rangle$ acts on $U$. Observe that, for $v \in U$, we have $\pi A_{v}=\left\{x \in U^{*} \mid\right.$ $\langle x, v\rangle>0\}$. On the other hand, if $|I|=2$, the proposition is readily seen to hold. Hence $\left.\pi A_{v} \cap w\right|_{U} ^{*} \pi A_{v} \neq \emptyset$ implies $w=1$. Suppose now $\left.f \in A_{i j} \cap w\right|_{U} ^{*} A_{i j}$. Then both $\left.f\right|_{U} \in \pi A_{i j}$ and $\left.\left.f\right|_{U} \in \pi w\right|_{U} ^{*} A_{i j}$, whence $\left.f\right|_{U} \in \pi\left(A_{w e_{i}} \cap A_{w e_{j}}\right)=\pi A_{w e_{i}} \cap \pi A_{w e_{j}}=\left.w\right|_{U} ^{*} \pi A_{i j}$. Thus $\left.\left.f\right|_{U} \in \pi A_{i j} \cap w\right|_{U} ^{*} \pi A_{i j}$, giving $w=1$.

QED

We proceed to prove Theorem 4.1.

4.6 (i) $\Rightarrow$ (ii) For $r \in R$, take $e_{r}$ to be the norm 2 eigenvector of $r$ in $A_{r}$, and $\Phi=\bigcup_{r \in R} W e_{r}$. Setting $v>0$ if and only if $e_{s}^{*} \in A_{v}$ for each $s \in R$, we obtain $w A \subseteq A_{r}$ if and only if $w^{-1} e_{r}>0$. This gives the first two properties of a root system as in (ii). If $\alpha \in \Phi \backslash\left\{e_{r}\right\}$ and $\alpha>0$, then $\left\langle r(\alpha), e_{s}\right\rangle=\left\langle\alpha, e_{s}\right\rangle>0$ for at least one $s \neq r$, so $r(\alpha)>0$, whence the third property of a root system. Finally, suppose $w e_{r}=e_{s}$ for certain $r, s \in R$ and $w \in W$. 
Then, for any $v \in V$,

$$
\begin{aligned}
w r w^{-1} s(v) & =w r\left(w^{-1} v-B\left(v, e_{s}\right) w^{-1} e_{s}\right) \\
& =w\left(w^{-1} v-B\left(v, e_{s}\right) e_{r}-B\left(w^{-1} v-B\left(v, e_{s}\right) e_{r}, e_{r}\right) e_{r}\right) \\
& =v-B\left(v, e_{s}\right) w e_{r}-B\left(w^{-1} v-B\left(v, e_{s}\right) e_{r}, e_{r}\right) w e_{r} \\
& =v-B\left(v, e_{s}\right) w e_{r}-\left(B\left(w^{-1} v, e_{r}\right)+2 B\left(v, e_{s}\right)\right) w e_{r} \\
& =v
\end{aligned}
$$

proving $w r w^{-1}=s$. Hence (ii).

4.7 (ii) $\Rightarrow$ (iii) First of all note that $r e_{r}=-e_{r}$. This follows from the second condition in (ii) as $\alpha=-r e_{r} \in \Phi$ satisfies both $\alpha>0$ and $r \alpha<0$.

Second, note that we may replace $\Phi$ by $\bigcup_{r \in R} W e_{r}$. We shall do so and show that then $T$ and $\Phi$ are equivalent $W$-sets. To this end, assign to $t=w r w^{-1} \in T$, with $r \in R$ and $w \in W$, the element $e_{t} \in\left\{ \pm w e_{r}\right\}$ with $e_{t}>0$. To see that $e_{t}$ is well defined, suppose we also have $t=w^{\prime} r^{\prime} w^{\prime-1}$ for $r^{\prime} \in R$ and $w^{\prime} \in W$. Then $r w^{-1} w^{\prime}=w^{-1} w^{\prime} r^{\prime}$, leading to $r\left(w^{-1} w^{\prime} e_{r^{\prime}}\right)=w^{-1} w^{\prime} r^{\prime} e_{r^{\prime}}=-\left(w^{-1} w^{\prime} e_{r^{\prime}}\right)$, so that the root system properties yield $w^{-1} w^{\prime} e_{r^{\prime}} \in\left\{ \pm e_{r}\right\}$; thus $w^{\prime} e_{r^{\prime}}$ and $w e_{r}$, both being $>0$, must coincide. The inverse of the map $t \mapsto e_{t}$ from $T$ to $\Phi$ is given by $w e_{r} \mapsto w r w^{-1}(r \in R ; w \in W)$; it is well defined in view of the last property of root systems. Thus $t \mapsto e_{t}$ is a bijection, which is readily seen to be $W$-equivariant.

Now suppose $w=r_{1} \cdots r_{q}$ and $t \in T$. If $w^{-1} e_{t}<0$, then, as $e_{t}>0$, there exists $i \in$ $\{1, \ldots, q\}$ such that $r_{i-1} \cdots r_{1} e_{t}>0$ and $r_{i} \cdots r_{1} e_{t}<0$. But then the root system properties imply $r_{i-1} \cdots r_{1} e_{t}=e_{r_{i}}$, whence, by the above equivalence, $t=r_{1} \cdots r_{i-1} r_{i} r_{i-1} \cdots r_{1}$, so $t w=r_{1} \cdots r_{i-1} \widehat{r_{i}} r_{i+1} \cdots r_{q}$. We have shown:

$\left(^{*}\right)$ if $w=r_{1} \cdots r_{q}$ and $t \in T$ satisfy $w^{-1} e_{t}<0$, then $t w=r_{1} \cdots r_{i-1} \widehat{r_{i}} r_{i+1} \cdots r_{q}$ for some

$i$.

Define $\nu: W \rightarrow \mathbb{Z} /(2) T$ by

$$
\nu(w)=\sum_{t \in T, w^{-1} e_{t}<0} t \quad(w \in W) .
$$

Observe that, by $\left(^{*}\right)$, the cardinality of $\left\{t \in T \mid w^{-1} e_{t}<0\right\}$ equals $l(w)$; in particular it is finite so $\nu$ is well defined. By the second and third property of (ii), we see $\nu(r)=r$ for $r \in R$. Finally, for $v, w \in W$, we have $\nu(v)=\sum\left\{t \in T \mid v^{-1} e_{t}<0\right.$ and $\left.w^{-1} v^{-1} e_{t}<0\right\}$ $+\sum\left\{t \in T \mid v^{-1} e_{t}<0\right.$ and $\left.w^{-1} v^{-1} e_{t}>0\right\}$, while $v \circ \nu(w)=\sum\left\{t \in T \mid v^{-1} e_{t}>0\right.$ and $\left.w^{-1} v^{-1} e_{t}<0\right\}+\sum\left\{t \in T \mid v^{-1} e_{t}<0\right.$ and $\left.w^{-1} v^{-1} e_{t}>0\right\}$, so that $\nu(v)+v \circ \nu(w)=\sum\{t \in$ $\left.T \mid w^{-1} v^{-1} e_{t}<0\right\}=\nu(v w)$. Hence (iii).

4.8 (iii) $\Rightarrow$ (iv) For $t \in T$ and $w \in W$, let $\nu_{t}(w) \in\{0,1\}$ be such that $\nu(w)=\sum_{t \in T} \nu_{t}(w) t$. We claim $l(w)=\sum_{t \in T} \nu_{t}(w)$. To establish the claim, suppose $r_{1} \cdots r_{q}$ is a reduced expression for $w \in W$ (so that $q=l(w))$. Then $\nu(w)=t_{1}+\ldots+t_{q}$, where $t_{i}=\left(r_{1} \cdots r_{i-1}\right) \circ r_{i}$. If $t_{i}=t_{j}$ for $i<j$, then $\left(r_{i} \cdots r_{j-1}\right) \circ r_{j}$ whence $w=r_{1} \cdots \widehat{r_{i}} \cdots \widehat{r_{j}} \cdots r_{q}$, contradicting $l(w)=q$. Hence the claim. Note also, for future use, that the above also gives: $\nu_{t}(w)=1$ implies $l(t w)<l(w)$. Next we show that $\nu_{t}(t)=1$ for any $t \in T$. To this end, write $t=\left(r_{m} \cdots r_{1}\right) \circ r_{0}$. If $m=0$ then $t \in R$ and there is nothing to show. Suppose therefore, 
$m>0$. Then $\nu(t)=\nu\left(r_{m}\left(\left(r_{m} t r_{m}\right) r_{m}\right)\right)=\nu\left(r_{m}\right)+r_{m} \circ\left(\nu\left(r_{m} t r_{m}\right)+\left(r_{m} t r_{m}\right) \circ \nu\left(r_{m}\right)\right)=$ $r_{m}+r_{m} \circ \nu\left(r_{m} \circ t\right)+t \circ r_{m}$. Since $t \neq r_{m}$, this yields $\nu_{t}(t)=\nu_{r_{m} \circ t}\left(r_{m} \circ t\right)$ and, as $r_{m} \circ t=\left(r_{m-1} \cdots r_{1}\right) \circ r_{0}$, we are done by induction on $m$.

From this identity, we see $\nu_{t}(t w) \equiv \nu_{t}(t)+\nu_{t}(w) \equiv 1+\nu_{t}(w) \bmod 2$. Thus $\nu_{t}(w)=0$ gives $\nu_{t}(t w)=1$. Recall that that $\nu_{t}(w)=1$ implies $l(t w)<l(w)$. Application of this statement to $t w$ instead of $w$ gives that $\nu_{t}(t w)=1$ implies $l(w)<l(t w)$ (observe that $t t w=w)$. We have reached the dichotomy: either $\nu_{t}(w)=1$ and $l(t w)<l(w)$, or $\nu_{t}(w)=0$ and $l(w)<l(t w)$. Now (iv) is immediate.

4.9 (iv) $\Rightarrow(v)$ For $w \in W$ let $\operatorname{Bruh}(w)$ be the set of all elements in $W$ of the form $t_{q} \cdots t_{1} w$ (for some natural number $q$ ) with $l\left(t_{i} \cdots t_{1} w\right) \leq l\left(t_{i-1} \cdots t_{1} w\right)$ for each $i \in\{1, \ldots, q\}$. By the Strong Exchange Condition, the elements of $\operatorname{Bruh}(w)$ are of the shape required in the first condition of (v). The converse needs more elaboration. Suppose $w=r_{1} \cdots r_{q}$ is a reduced expression for $w$ and consider the element $z$ obtained by deletion of $r_{i_{1}}, \ldots, r_{i_{m}}$ for certain $1 \leq i_{1}<\cdots<i_{m} \leq q$. If $m=0$ then $z=w \in \operatorname{Bruh}(w)$. Therefore, assume $m>0$. Put $t=r_{1} \cdots r_{i_{1}-1} r_{i_{1}} r_{i_{1}-1} \cdots r_{1}$, so that $t z$ is obtained from $w$ by deletion of $r_{i_{2}}, \ldots, r_{i_{m}}$. By induction on $m$, we may assume $t z \in \operatorname{Bruh}(w)$. Thus we are done if $l(t z) \geq l(z)$. Assume therefore $l(t z)<l(z)$. Then, by the Strong Exchange Condition, there is $j \in\{1, \ldots, q\} \backslash\left\{i_{1}, \ldots, i_{m}\right\}$ such that $t z$ is obtained from $w=r_{1} \cdots r_{q}$ by deletion of $r_{j}, r_{i_{1}}, \ldots, r_{i_{m}}$. If $j<i_{1}$, then $r_{1} \cdots \widehat{r_{j}} \cdots r_{i_{1}-1}=r_{1} \cdots r_{i}$, contradicting that $w=r_{1} \cdots r_{q}$ is a reduced expression for $w$. Hence $j>i_{1}$. Moreover, $z=t(t z)$ can be obtained from $w=r_{1} \cdots r_{q}$ by deletion of $r_{i_{2}}, \ldots, r_{j}, \ldots, r_{i_{m}}$. Thus, for fixed $m$, we have reduced to a case in which the subsequence of deleted generators occurs 'higher up' (in terms of indices). The highest case $z=r_{1} \cdots r_{q-m}$ being obvious, this proves by recursion that $z \in \operatorname{Bruh}(w)$, always.

It remains to show that, for any $t \in T$ and $w \in W$, we have $w \in t \operatorname{Bruh}(w) \cup \operatorname{Bruh}(t w)$. From the above definition of the map Bruh, it is clear that either $l(t w)<l(w)$ and $t w \in$ $\operatorname{Bruh}(w)$ or $l(t(t w))<l(t w)$ and $w=t(t w) \in \operatorname{Bruh}(t w)$, whence the result.

$4.10(\mathrm{v}) \Rightarrow$ (vi) For each $r \in R$, set $C_{r}=\{w \in W \mid l(w)<l(r w)\}$. Then, clearly $1 \in C_{r}$, and, more generally, $w \in C_{r}$ if and only if $w \in \operatorname{Bruh}(r w)$. Also $C_{r} \cap r C_{r}=\emptyset$ is immediate. Suppose $w \in W$ and $r, r^{\prime} \in R$ satisfy $w \in C_{r} \backslash C_{r} r^{\prime}$. Then $w r^{\prime} \notin \operatorname{Bruh}\left(r w r^{\prime}\right)$, so $w r^{\prime} \in$ $r \operatorname{Bruh}\left(w r^{\prime}\right)$, proving $l\left(r w r^{\prime}\right)<l\left(w r^{\prime}\right)$. Consequently, $l\left(w r^{\prime}\right)=l\left(r w r^{\prime}\right)+1 \geq l(r w)>l(w)$. So, from a reduced expression $r_{1} \cdots r_{q}$ for $w$, we obtain the reduced expression $r_{1} \cdots r_{q+1}$ for $w r^{\prime}$ with $r_{q+1}=r^{\prime}$. From $r w r^{\prime} \in \operatorname{Bruh}\left(w r^{\prime}\right)$, we obtain $j \in\{1, \ldots, q+1\}$ with $r w r^{\prime}=r_{1} \cdots \widehat{r_{j}} \cdots r_{q+1}$. But $j \leq q$ would contradict $l(r w)>l(w)$, so $j=q+1$, proving $r w r^{\prime}=w$; this establishes $r^{\prime}=w^{-1} r w$, as required.

4.11 (vi) $\Rightarrow$ (vii) Suppose $w \in W \backslash C_{r}$. Let $r_{1} \cdots r_{q}$ be a reduced expression for $w$. Since $1 \in C_{r}$ and $w \notin C_{r}$, there must be $j \in\{1, \ldots, q\}$ with $r_{1} \cdots r_{j-1} \in C_{r} \backslash C_{r} r_{j}$. By the last property of (vi), this gives $r_{j-1} \cdots r_{1} r r_{1} \cdots r_{j-1}=r_{j}$, whence $r w=r_{1} \cdots \widehat{r_{j}} \cdots r_{q}$ and $l(r w)<l(w)$. Thus $\{w \in W \mid l(w)<l(r w)\} \subseteq C_{r}$. But then $\{w \in W \mid l(w)>l(r w)\}=$ $r\{w \in W \mid l(w)<l(r w)\} \subseteq r C_{r}$, and $C_{r} \cap r C_{r}=\emptyset$ gives $C_{r}=\{w \in W \mid l(w)<l(r w)\}$.

We now derive the Exchange Condition. Let $r_{1} \cdots r_{q}$ be a reduced expression for $w \in W$ and suppose $l(r w)<l(w)$ for some $r \in R$. Then $w \notin C_{r}$, so, by the above, there exists $j \in\{1, \ldots, q\}$ with $r w=r_{1} \cdots \widehat{r_{j}} \cdots r_{q}$, proving (vii).

The proof that (vii) implies that $(W, R)$ is a Coxeter system uses the following remarkable result. 
4.12 Lemma Suppose $(W, R)$ satisfies the Exchange Condition. Let $M$ be the matrix over $R$ (assuming some ordering on $R$ ) whose $r$, s-entry $m_{r, s}$ is the order of $r s$. If $F$ is a monoid (with unit) affording a map $\phi: R \rightarrow F$ such that for any two distinct $r, s \in R$ we have

$$
\phi(r) \phi(s) \phi(r) \cdots\left[m_{r, s} \text { factors }\right]=\phi(s) \phi(r) \phi(s) \cdots\left[m_{r, s} \text { factors }\right] \text { if } m_{r, s}<\infty
$$

then $\phi$ can be extended to a mapping, also called $\phi$, from $W$ to $F$ such that $\phi(w)=\phi r_{1} \cdots \phi r_{q}$ whenever $r_{1} \cdots r_{q}$ is a reduced expression for $w$.

Proof We shall work in the free monoid $I^{*}$ on a copy $I$ of $R$. The identification of $I$ and $R$ will be made through a map $\rho: I \rightarrow R$ which naturally extends to a morphism $\rho: I^{*} \rightarrow W$ of monoids. Let $I_{w}^{*}$ be the subset of $I^{*}$ consisting of all minimal words for $w$, that is all $\mathrm{i} \in I^{*}$ of length $l(w)$ with $\rho(\mathbf{i})=w$. Given $\mathbf{i}=i_{1} \cdots i_{q} \in I^{*}$, we put $\phi(\mathbf{i})=\phi\left(i_{1}\right) \cdots \phi\left(i_{q}\right)$. We want to show that $\phi(\mathbf{i})=\phi\left(\mathbf{i}^{\prime}\right)$ for all $\mathbf{i}, \mathbf{i}^{\prime} \in I_{w}^{*}$. We proceed by induction on $l(w)$. If $l(w)=1$, the Exchange Condition yields that $\left|I_{w}^{*}\right|=1$, so there is nothing to prove. Assume $l(w)>1$, and let $\mathrm{i}=i_{1} \cdots i_{q}$ and $\mathrm{i}^{\prime}=i_{1}^{\prime} \cdots i_{q}^{\prime}$ be two minimal words for $w$. Put $i=i_{1}^{\prime}$. We have $l(\rho(i) w) \leq q-1$, so the Exchange Condition gives $\rho\left(i i_{1} \cdots i_{j-1}\right)=\rho\left(i_{1} \cdots i_{j}\right)$ for some $j \leq q$. Thus, $\mathbf{i}^{\prime \prime}=i i_{1} \cdots i_{j-1} i_{j+1} \cdots i_{q} \in I_{w}^{*}$. Deleting the first letters of $\mathbf{i}^{\prime}$ and $\mathbf{i}^{\prime \prime}$ and applying induction to $\rho(i) w$, we obtain $\phi\left(\mathbf{i}^{\prime}\right)=\phi\left(\mathbf{i}^{\prime \prime}\right)$. If $j<q$, then, comparing the last terms of $\mathbf{i}^{\prime \prime}$ and $\mathbf{i}$ and applying induction to $w \rho\left(i_{q}\right)$, we obtain $\phi(\mathbf{i})=\phi\left(\mathrm{i}^{\prime \prime}\right)$, so that we are done.

Therefore, we may assume $j=q$. Then, replacing the pair $\mathrm{i}, \mathrm{i}^{\prime}$ by $\mathrm{i}^{\prime \prime}, \mathrm{i}$, and using the same arguments, we obtain $\mathbf{i}^{\prime \prime \prime}=i_{1} i_{1} \cdots i_{q-2} \in I_{w}^{*}$ with $\phi\left(\mathbf{i}^{\prime \prime \prime}\right)=\phi(\mathbf{i})$. Repeating this process, we obtain $\mathrm{u}=i_{1} \ddot{i}_{1} \cdots$ and $\mathrm{v}=i i_{1} i \cdots \in I_{w}^{*}$, each sequence involving only $i$ and $i_{1}$ alternately, with $\phi(\mathbf{u})=\phi(\mathbf{i})$ and $\phi(\mathbf{v})=\phi\left(\mathbf{i}^{\prime}\right)$. Now $\rho(\mathbf{u})=\rho(\mathbf{v})$ and $l(\mathbf{u})=l(\mathbf{v})=q$ imply that $q$ is the order of $\rho(i) \rho\left(i_{1}\right)$, so the hypothesis of the lemma gives $\phi(\mathrm{u})=\phi(\mathrm{v})$, whence $\phi(\mathrm{i})=\phi\left(\mathrm{i}^{\prime}\right)$. Thus the mapping $\phi$ is constant on each $I_{w}^{*}$, so its restriction to $\bigcup_{w \in W} I_{w}^{*}$ factors through $W$ as required.

4.13 (vii) implies that $(W, R)$ is a Coxeter system Let $M$ be the matrix over $R$ as given in the lemma. Denote by $(\bar{W}, \bar{R})$ the Coxeter system of type $M$. We shall apply the lemma to the canonical mapping $r \mapsto \bar{r}$ from $R$ to $\bar{R}$, taking $F$ to be the monoid underlying the group $\bar{W}$. By definition of $(\bar{W}, \bar{R})$, this mapping satisfies the hypothesis of the lemma. Hence we obtain a mapping $w \mapsto \bar{w}$ from $W$ to $\bar{W}$ such that $\bar{w}=\bar{r}_{1} \cdots \bar{r}_{q}$ whenever $w=r_{1} \cdots r_{q}$ and $q=l(w)$. We claim that $w \mapsto \bar{w}$ is a homomorphism. First, we show that $\overline{r w}=\overline{r w}$ for all $r \in R, w \in W$. If $l(r w)=q+1$, we have $\overline{r w}=\bar{r} \bar{r}_{1} \cdots \bar{r}_{q}=\bar{r} \bar{w}$. If $l(r w) \leq q$, the Exchange Condition gives a $j \in\{1, \ldots, q\}$ with $r w=r_{1} \cdots r_{j-1} r_{j+1} \cdots r_{q}$, whence $l(r w)=q-1$. By use of $\bar{r}_{j}^{2}=1$, we obtain

$$
\begin{aligned}
\overline{r w} & =\bar{r}_{1} \cdots \bar{r}_{j-1} \bar{r}_{j+1} \cdots \bar{r}_{q}=\bar{r}_{1} \cdots \bar{r}_{j-1} \bar{r}_{j}^{2} \bar{r}_{j+1} \cdots \bar{r}_{q} \\
& =\bar{r} \bar{r}_{1} \cdots \bar{r}_{j-1} \bar{r}_{j} \bar{r}_{j+1} \cdots \bar{r}_{q}=\bar{r} \bar{w},
\end{aligned}
$$

Next, we settle $\overline{u v}=\bar{u} \bar{v} \bar{r}$ for all $u, v \in W$, by induction on $l(u)$. The case $l(u)=1$ has just been treated. Assume $l(u)>1$. Then $u=r u^{\prime}$ for some $r \in R, u^{\prime} \in W$ with $l\left(u^{\prime}\right)<l(u)$, so

$$
\overline{u v}=\overline{r\left(u^{\prime} v\right)}=\bar{r} \overline{u^{\prime} v}=\bar{r} \overline{u^{\prime}} \bar{v}=\left(\bar{r} \overline{u^{\prime}}\right) \bar{v}=\bar{u} \bar{v},
$$


proving that $w \mapsto \bar{w}$ is a morphism indeed. Finally the morphism is clearly surjective, and, since $\bar{W}$ is freely generated by the relations $(\bar{r} \bar{s})^{m_{r, s}}=1(r, s \in R)$, it must be an isomorphism.

\section{Beautiful properties}

Here we discuss a number of properties of Coxeter groups which follow more or less straightforwardly from the characterizations in the previous section. Proofs are to be found in [Bourb 1968] and [BCN 1989]. First of all note that, due to Corollary 4.3(i), the sets $I$ and $R$ can be identified via $\rho$. We shall still use $\rho$ if confusion is imminent, e.g., when discussing the word problem.

Fix a Coxeter system $(W, R)$ and let $J, K$ be subsets of $R$. The subgroup $\langle J\rangle$ of $W$, also denoted by $W_{J}$, is the subgroup generated by $J$. It is again a Coxeter group, as we shall soon see. At any rate, $W_{\emptyset}=\{1\}$ and $W_{R}=W$, and $J \subseteq K$ implies $W_{J} \subseteq W_{K}$. Set

$$
D_{J, K}=\{w \in W \mid l(r w)=l(w s)>l(w) \text { for all } s \in J \text { and } r \in K\} \text {. }
$$

The set $D_{J, K}$ will turn out to be a natural system of double $W_{J}, W_{K}$-coset representatives.

5.1 Proposition Let $(W, R)$ be a Coxeter system, and let $J, K$ be subsets of $R$.

(i) Suppose $r_{1} r_{2} \cdots r_{q}$ is a non-reduced expression for $w \in W$. Then there are indices $i, j \in\{1, \ldots, q\}$ such that $r_{1} r_{2} \cdots r_{q}=r_{1} \cdots r_{i-1} r_{i+1} \cdots r_{j-1} r_{j+1} \cdots r_{q}$. Thus, given a word in $\rho^{-1}(w)$, a minimal word for $w$ can be found by repeatedly cancelling factors in pairs.

(ii) For each $w \in W$ there is a subset $R_{w}$ of $R$ such that $R_{w}=\left\{r_{1}, \ldots, r_{q}\right\}$ for each reduced expression $w=r_{1} \cdots r_{q}$.

(iii) If $w \in W_{J}$, then $l(w)=l_{J}(w)$ and $R_{w} \subseteq J$.

(iv) The map $\phi_{J, K}: D_{J, K} \rightarrow W_{J} \backslash W / W_{K}$ sending $w \in D_{J, K}$ to $W_{J} w W_{K}$ is a bijection. Moreover, $D_{J, K}=D_{J, \emptyset} \cap D_{\emptyset, K}$. Each $w \in W$ has a reduced expression $w=u d v$ with $d \in D_{J, K}, u \in W_{J}, v \in W_{K}$. In particular, $\phi_{J, K}^{-1}\left(W_{J} w W_{K}\right)$ consists of the unique shortest element of $W_{J} w W_{K}$.

(v) If $W$ is finite, there is a unique longest element $w_{0}$ in $W$. This element is an involution with $w_{0} R w_{0}=R$.

(vi) If $\gamma$ is the Reflection Representation, then $W_{J}=\left\{w \in W \mid \gamma^{*}(w) e_{i}^{*}=e_{i}^{*}\right.$ for each $i \in R \backslash J\}$.

Proof (i) is immediate from the Exchange Condition.

(ii) We apply Lemma 4.12 to the map $r \mapsto\{r\}$ from $R$ to the monoid $\mathcal{P}(R)$ of all subsets of $R$ in which multiplication is given by set theoretic union (the empty set is the unit). Since $\{r\} \cup\{s\} \cup\{r\} \cup \cdots=\{r, s\}$, the hypothesis of the lemma is satisfied. Therefore, the map can be extended to a map $w \mapsto R_{w}$ such that $R_{w}=\left\{r_{1}, \ldots, r_{q}\right\}$ for every reduced expression $r_{1} \cdots r_{q}$ of $w$.

(iii) The first part of (iii) is obvious from (i). As for the second, let $r_{1} \cdots r_{q}$ be a reduced expression for $w$, then $r_{q} \cdots r_{1}$ is a reduced expression for $w^{-1}$, so, by (ii), $R_{w}=R_{w^{-1}}$ Furthermore, by the Exchange Condition $R_{r w} \subseteq\{r\} \cup R_{w}$, so $R_{v w} \subseteq R_{v} \cup R_{w}$ for all 
$v, w \in W$. Hence $\left\{w \in W \mid R_{w} \subseteq J\right\}$ is a subgroup of $W_{J}$ containing $J$. Consequently, $W_{J}=\left\{w \in W \mid R_{w} \subseteq J\right\}$.

For (iv) and (v), see [Bourb 1968, Ch. IV, Ex. 1.3 and Ch. IV, Ex. 1.22].

(vi) Clearly, each element of $W_{J}$ fixes all $e_{i}^{*}$ for $i \in I \backslash J$. Conversely, suppose $\rho\left(i_{1}\right) \cdots \rho\left(i_{q}\right)$ is a reduced expression of $w \in W$ with $w e_{i}^{*}=e_{i}^{*}$ (again, we suppress $\gamma$ in the notation). Then, by $4.1(\mathrm{ii}), 0 \leq\left\langle e_{i}^{*}, e_{i_{q}}\right\rangle=\left\langle e_{i}^{*}, w e_{i_{q}}\right\rangle \leq 0$, establishing that $i \neq i_{q}$ so that $\rho\left(i_{q}\right) e_{i}^{*}=e_{i}^{*}$. Thus we can finish by induction applied to $\rho\left(i_{1}\right) \cdots \rho\left(i_{q-1}\right)$, to show that $\rho_{i} \notin R_{w}$. This gives (vi).

QED

Some of the distributive laws only the freshman would dream of hold for certain natural subgroups of Coxeter groups:

5.2 Theorem (Convexity) Let $W$ be a Coxeter group over $M=\left(m_{r s}\right)_{r, s \in R}$, and suppose $J, K, L$ are subsets of $R$. Then

(i) $\left(W_{J}, J\right)$ is a Coxeter system of type $\left.M\right|_{J \times J}$.

(ii) $W_{J} \cap W_{K}=W_{J \cap K}$.

(iii) $W_{J} W_{K} \cap W_{L}=\left(W_{J} \cap W_{L}\right)\left(W_{J} \cap W_{L}\right)$.

(iv) $W_{J}\left(W_{K} \cap W_{L}\right)=\left(W_{J} W_{K}\right) \cap\left(W_{J} W_{L}\right)$.

(v) If $w \in D_{J, K}$ then $W_{J} \cap w W_{K} w^{-1}=\left\langle J \cap w K w^{-1}\right\rangle$.

(vi) If $w \in D_{J, J}$ and $x, y \in W$ satisfy $x w=w y$ then $x \in\left\langle R_{w}\right\rangle\left\langle R_{w}^{\perp}\right\rangle$, where $K^{\perp}=\{r \in R \mid$ $r s=s r$ for all $s \in K\}$.

(vii) If $l(s x)<l(x)$ and $l(s y)<l(y)$ then $l\left(x^{-1} s y\right)>l\left(x^{-1} y\right)$

Proof (i) If $w \in W_{J}$, then by (iii) of the above proposition, the length of $w$ in $(W, R)$ and in $\left(W_{J} J\right)$ are equal. Therefore, the Exchange Condition in $(W, R)$ implies the Exchange Condition in $\left(W_{J}, J\right)$. By Theorem 4.1, the latter must be a Coxeter system.

(ii) If $w \in W_{J} \cap W_{K}$, then by (i) the set $R_{w}$ is contained in both $J$ and $K$. Hence $w \in W_{J \cap K}$, and $W_{J} \cap W_{K} \subseteq W_{J \cap K}$. The converse inclusion is obvious.

(iii), (iv) can be shown to hold similarly to (ii). See [BCN 1989] for a proof of (v) and (vi).

(vii) Let $x, x_{1}, \ldots, x_{l}=s y$ be a minimal path in $\mathcal{C}(W, R)$ from $x$ to $s y$. Thus, $l=l\left(x^{-1} s y\right)$. Recall the definition of $C_{r}$ from 4.1(vi). Since $x \in s C_{s}$ and $s y \in C_{s}$ (as $l(s x)<l(x)$ and $l(s y)<l(y))$, we have $m \in\{0, \ldots, l-1\}$ such that $x_{m} \in s C_{s}$ and $x_{m+1} \in C_{s}$. From the existence of $r \in R$ with $x_{m+1}=x_{m} r$, we see $s x_{m} \in C_{s} \backslash C_{s} r$. By $4.1(\mathrm{vi}), x_{m+1}=x_{m} r=$ $s x_{m}$, so $x=x_{0}, x_{1}, \ldots, x_{m}=s x_{m+1}, s x_{m+2}, \ldots, s x_{l}=y$ is a path in $\mathcal{C}(W, R)$ from $x$ to $y$ of length $l-1$. Consequently, $l\left(x^{-1} s y\right)>l\left(x^{-1} y\right)$.

QED

In terms of chamber systems, (ii) represents a convexity property whereas (iii) and (iv) are known as intersection properties. (vii) will be used in the proof of 7.10.

Property (i) of 5.1 can be further refined. Write $\mathrm{k}_{i, j}$ for the word $i j i \cdots \in I^{*}$ of length $m_{i, j}$. Thus $\rho\left(\mathbf{k}_{i, j}\right)=\rho\left(\mathbf{k}_{j, i}\right)$. If $\mathbf{i}, \mathbf{j} \in I^{*}$, set $\mathbf{i} \sim \mathbf{j}$ if there are $\mathbf{a}, \mathbf{b} \in I^{*}$ and $r, s \in I$ such that $\mathbf{i}=\mathrm{ak}_{r, s} \mathrm{~b}$ and $\mathrm{j}=\mathbf{a k}_{s, r} \mathrm{~b}$. Recall the definition of $I_{w}^{*}$ from the proof of Lemma 4.12. Clearly $I_{w}^{*}$ is a union of connected components of the graph $\left(I^{*}, \sim\right)$.

5.3 Theorem ([Tits 1969]) Let $(W, R)$ be a Coxeter system and $w \in W$. Then

(i) $I_{w}^{*}$ is a connected component of the graph $\left(I^{*}, \sim\right)$. 
(ii) If $X$ is a connected component distinct from $I_{\rho(\mathrm{j})}^{*}$ where $\mathrm{j} \in X$, then there exists $\mathbf{i}=i_{1} \cdots i_{q} \in X$ with $i_{j}=i_{j+1}$ for some $j \in\{1, \ldots, q-1\}$.

(iii) If $w \in W$ and $i, j \in I$ satisfy $l\left(w \rho_{i}\right)=l\left(w \rho_{j}\right)<l(w)$, then there exists $\mathbf{x} \in I^{*}$ such that $\mathrm{xk}_{i, j} \in I_{w}^{*}$.

5.4 Reflections Elements of the set $T=\left\{w r w^{-1} \mid r \in R, w \in W\right\}$ are called reflections. Theorem 5.2(i) shows that certain very special subgroups of $W$ generated by reflections are again Coxeter groups. Much more generally, let $U$ be a reflection subgroup, that is, a subgroup generated by reflections (so $U=\langle U \cap T\rangle$ ). Set

$$
R_{U}=\left\{t \in T \cap U \mid \text { if } t^{\prime} \in T \cap U \text { with } l\left(t^{\prime} t\right)<l(t) \text { then } t^{\prime}=t\right\} .
$$

Then, according to Deodhar and Dyer (cf. [Deo 1989] and [Dyer 1990]):

5.5 Theorem Let $(W, R)$ be a Coxeter system and $U$ a reflection subgroup. Retain the above notation for $T$ and $R_{U}$. Then $\left(U, R_{U}\right)$ is a Coxeter system and $T \cap U$ is the set of all reflections of $U$.

The proof is based on:

\subsection{Lemma}

(i) $R \cap U \subseteq R_{U}$;

(ii) let $r \in R$; if $r \in U$ then $R_{r U r}=R_{U}$; otherwise, $R_{r U r}=r \circ R_{U}$;

(iii) If $t \in U \cap T$ there are $t_{0}, \ldots, t_{m} \in R_{U}$ with $t=\left(t_{m} \cdots t_{1}\right) \circ t_{0}$;

(iv) the map $\nu_{U}: W \rightarrow \bigoplus_{t \in T \cap U} \mathbb{Z} /(2) t$ given by $\nu_{U}(w)=\nu(w) \cap U$ satisfies $\nu_{U}(v w)=$ $\nu_{U}(v)+v \circ \nu_{U}(w)$ for all $v \in W, w \in U$

Here the operator $\cap U$ on $\bigoplus_{t \in T} \mathbb{Z} /(2) t$ is the obvious projection onto $\bigoplus_{t \in T \cap U} \mathbb{Z} /(2) t$.

Proof First observe that $R_{U}=\left\{t \in T \mid \nu_{U}(t)=t\right\}$. Let $r \in R$.

(i) If $r \in R \cap U$, then $\nu_{U}(r)=r \cap U=r$ so $r \in R_{U}$.

(ii) The first case is immediate from $r U r=U$ if $r \in U$. Suppose $r \notin U$ and $t \in R_{U}$. Then $\nu(r t r)=r+r \circ \nu(t r)=r+r \circ \nu(t)+r \circ(t \circ r)=r+r \circ \nu(t)+(r t) \circ r$. As $r \notin U$, we also have $r \notin r \circ U$ and $(r t) \circ r \notin r \circ U$. Thus $\nu(r t r) \cap r \circ U=r \circ \nu(t) \cap r \circ U=r \circ(\nu(t) \cap U)=r \circ \nu_{U}(t)$. Hence $r \circ R_{U} \subseteq R_{\text {roU }}$. Applying this result to $r \circ U$ instead of $U$, we obtain $r \circ R_{\text {roU }} \subseteq R_{U}$, whence $R_{r \circ U} \subseteq r \circ R_{U}$, proving (ii).

(iii) The proof is by induction on $l(t)$. If $l(t)=1$, then $t \in R \cap U \subseteq R_{U}$ by (i). Suppose $l(t)>$ 1 and take a reduced expression $r_{1} \cdots r_{2 m+1}$ for $t$ (recall that $l(t)$ is odd since this is true for a conjugate of $t$ in $R$ ). Then (by length consideration of $t^{-1} r_{1} \cdots r_{m+1}=r_{2 m+1} \cdots r_{m+2}$ ) we have $r_{2 m+1-i}=r_{i}$ for each $i=1, \ldots, m$, so that $t=\left(r_{1} \cdots r_{m}\right) \circ r_{m+1}$. Set $r=r_{1}$. As $r \circ t \in(r \circ U) \cap T$ has length $2 m-1$, the induction hypothesis provides for $t_{0}, \ldots, t_{k} \in R_{r \circ U}$ with $r \circ t=\left(t_{k} \cdots t_{1}\right) \circ t_{0}$. If $r \in U$, then $r \in r \circ U$ so, by (ii), $t=\left(r t_{k} \cdots t_{1}\right) \circ t_{0} \in R_{r \circ U}=R_{U}$. Otherwise, $r \notin r \circ U$, so setting $s_{i}=r \circ t_{i}$, we derive from (ii) $s_{i}=r \circ t_{i} \in r R_{r U r} r=R_{U}$, whence $t=\left(s_{k} \cdots s_{1}\right) \circ s_{0}$ as required.

(iv) is straightforward.

As for the proof of the above theorem, set

$$
T^{\prime}=\bigcup_{w \in\left\langle R_{U}\right\rangle} w R_{U} w^{-1}
$$


By (iii), $U \cap T \subseteq T^{\prime}$ so $U \cap T=T^{\prime}$. Furthermore,

$$
U=\langle U \cap T\rangle=\left\langle T^{\prime}\right\rangle \subseteq\left\langle R_{U}\right\rangle \subseteq U,
$$

whence $U=\left\langle R_{U}\right\rangle$. Now $\nu_{U}$ as defined in part (iv) of the above lemma is readily seen to satisfy the Cocycle Condition 4.1 (iii), so Theorem 4.1 yields that $\left(U, R_{U}\right)$ is a Coxeter system.

5.7 Example Consider the Coxeter system $(W,\{1,2,3\})$ of type $M$, where $M$ is the $3 \times 3$-matrix with all off-diagonal elements equal to $\infty$. Then the set $R_{6}=\{121,131,212$, $232,313,323\}$ of reflections in $W$ generates a reflection subgroup $U_{6}$. A direct check shows that $R_{U_{6}}=R_{6}$, so that $\left(U_{6}, R_{6}\right)$ is a Coxeter system of type a $6 \times 6$-matrix all of whose off-diagonal entries are $\infty$. Iterating this construction, it will be clear that one can obtain reflection subgroups of $W$ of arbitrary finite rank.

In the chamber system $\mathcal{C}(W, R)$, each reflection $t \in T$ corresponds to a wall, that is, the collection $\left\{\{w, w r\} \mid t=w r w^{-1}, w \in W, r \in R\right\}$ of edges of $\mathcal{C}(W, R)$. By use of the Hyperplane Condition 4.1.(vi), the roots $w e_{r}$ can also be interpreted in terms of the chamber system, see e.g. [Ron 1989].

5.8 The Bruhat order Let $(W, R)$ be a Coxeter system. From $4.1(\mathrm{v})$, it is clear that Bruh is uniquely determined by its properties. We shall write $\leq$ for the relation on $W$ defined by $x \leq w$ if $x \in \operatorname{Bruh}(w)$. Thus, $x \leq w$ if and only if there is a reduced expression $r_{1} \cdots r_{q}\left(r_{j} \in R\right)$ for $w$ such that $x=r_{i_{1}} \cdots r_{i_{m}}$ (where $1 \leq i_{1}<i_{2}<\ldots<i_{m} \leq q$ ). In view of Proposition 5.1(i) we may require that the expression for $x$ be reduced. Therefore, $\leq$ defines an order on $W$; it is the so-called Bruhat order. Clearly, 1 is the smallest element of $W$; in case $W$ is finite, the longest element $w_{0}$ (cf. $5.1(\mathrm{v})$ ) is the largest element.

See [Deo 1977], [Bjö 1984] and [BjWa 1982] for useful properties of the Bruhat order. We mention two, where $x, y \in W$ and $r \in R$ :

- If $l(r x) \leq l(x)$ and $l(r y) \leq l(y)$ then $x \leq y \Longleftrightarrow r x \leq y \Longleftrightarrow r x \leq r y$.

- If $x \leq y$ then there is a chain $x=x_{0} \leq x_{1} \leq \ldots \leq x_{t}=y \in W$ of elements of $W$ such that $l\left(x_{i-1}\right)=l\left(x_{i}\right)-1$ for all $i \in\{1, \ldots, t\}$. Moreover, if $x, y \in D_{\emptyset, J}$ for some $J \subseteq R$ then all $x_{i}$ can be chosen within $D_{\emptyset, J}$.

In [Deo 1987b], it is described how the Bruhat order can be recovered from its restriction to $D_{\emptyset, J}$ and an induced ordering on $W / W_{J}$ for $J \subseteq R$.

The Bruhat order is quite rigid: by [vdH 1974] (see also [Wate 1989]), up to group inversion, any Bruhat order automorphism on a Coxeter group of rank at least 3 is a group automorphism induced by a permutation of $R$ preserving $M$.

\section{On the structure of Coxeter groups}

Since each Coxeter group has a subgroup, denoted by $W^{+}$, of index 2 (cf. Example 3.1.(v)) there is only one simple Coxeter group (up to isomorphism); it has order 2 and $|R|=1$.

If the center $Z(W)$ of $W$ contains a non-identity element $w$, then $W$ is finite, and $w=w_{0}$ is the longest element of $W$, defined in 5.1(v). (See [BCN 1989] for a proof.)

In the case $W=W\left(A_{n}\right)$ for $n \geq 2$ the center is trivial and, for $n \geq 4$, the subgroup $W^{+} \cong$ Alt $_{n+1}$ is simple. The groups $W\left(I_{2}^{m}\right)$ and $W\left(F_{4}\right)$ are solvable, but if $n \geq 5$ and $W=W\left(B_{n}\right)$, then $Z(W) \leq W^{+}$and $W^{+} / Z(W) \cong 2^{n-1}$. Alt $t_{n}$ is neither solvable nor simple. 
6.1 Crystallographic groups Consider once more the Reflection Representation $\gamma$. A basic feature in Kac-Moody Lie algebra constructions, cf. [Kac 1985], is a $\gamma[W]$-invariant lattice $L$ in $\mathbb{R}^{n}$. The Coxeter group $(W, R)$ is called crystallographic if there is such a lattice $L$. Then, by study of $\mathbb{R} e_{i}+\operatorname{IR} e_{j}$, it follows that $m_{i, j} \in\{2,3,4,6, \infty\}$ for all $i, j \in I$. Another necessary condition is obtained by tracing a circuit $C$ in $(I, M)$ : the number of edges $\{i, j\}$ of $C$ with $m_{i, j} \equiv 0 \bmod 2$ and the number of edges $\{i, j\}$ of $C$ with $m_{i, j} \equiv 0 \bmod 4$ must both be even. Conversely, the three conditions on $M$ just stated, suffice for $(W, R)$ to be crystallographic.

A finite crystallographic group is called a Weyl group. All of the finite Coxeter groups we have seen thus far correspond to linear diagrams (and come from polytopes). The following non-linear diagrams lead to finite Weyl groups.

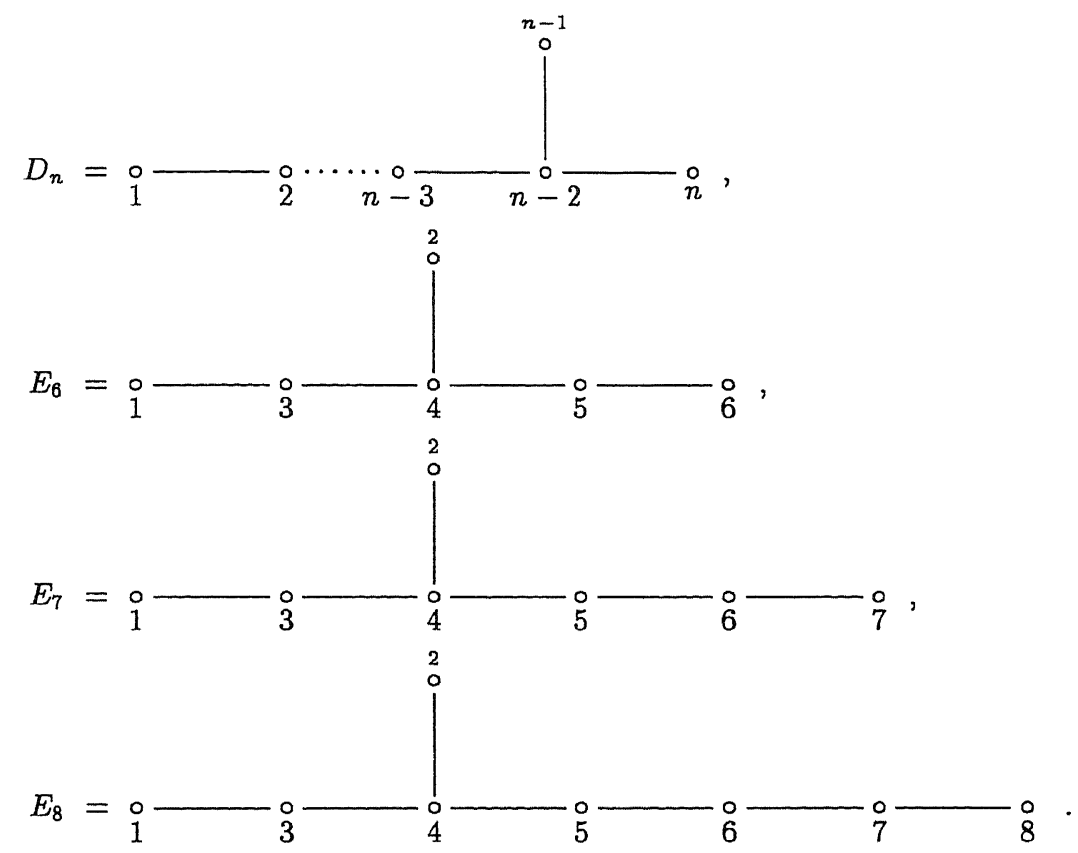

The easiest proof that $W(M)$ is finite for these $M$ is by checking that $B_{M}$ is positive definite and concluding that the root system $\Phi$, being a set of vectors in Euclidean space $\mathbb{R}^{n}$ with $(v, w) \in\{0, \pm 1, \pm 2\}$ for all $v, w \in \Phi$, must be finite. By Theorems 4.1 and 4.5, the permutation representation of $W$ on $\Phi$ is faithful, yielding that $W$ is finite.

There is a converse to this argument: the finite groups among Coxeter groups can be characterized as those for which the bilinear form $B$ of the Reflection Representation is positive definite.

The above diagrams are quite exceptional as may be clear from the following

6.2 Theorem (Finite Coxeter Groups) Let $(I, M)$ be a connected Coxeter diagram (cf. Example 3.1.(iv)). If the Coxeter group $W(M)$ is finite, then $M$ is one of $A_{n}(n \geq 3), B_{n}$ $(n \geq 3), D_{n}(n \geq 4), E_{n}(n=6,7,8), F_{4}, H_{n}(n=3,4), I_{2}^{m}(m \geq 3)$.

The Weyl groups among the finite reflection groups with connected diagram are $A_{n}$ $(n \geq 3), B_{n}(n \geq 3), D_{n}(n \geq 4), E_{n}(n=6,7,8), F_{4}$, and $G_{2}=I_{2}^{6}$. 
If $W$ is finite and $|R|=n$, the algebra of $W$-invariant polynomial functions on the Reflection Representation is isomorphic to the polynomial ring $\operatorname{IR}\left[X_{1}, \ldots, X_{n}\right]$. Conversely, if $W$ is a finite group of linear transformations on a real vector space $V$ and the algebra of $W$-invariant polynomial functions on that vector space is a polynomial ring then $W$ is a Coxeter group (cf. [Bourb 1968]). According to [Zales 1983], it is not true in general that the algebra of invariants of a group generated by reflections is a polynomial ring.

6.3 Affine Weyl groups and beyond The most famous series of infinite Coxeter groups are the Affine Weyl groups. These are the crystallographic groups for which the Reflection Representation is positive semidefinite. Their structure is $\mathbb{Z}^{n-1} \cdot W(M)$ for $W(M)$ a Weyl group of rank $n-1$. Conversely, for each Coxeter matrix $M$ of a finite Weyl group of rank $n-1$, there is an affine Weyl group of rank $n$. For $M=B_{n-1}(n \geq 4)$, there are two such affine Weyl groups, one is denoted by $\tilde{B}_{n-1}$, the other by $\tilde{C}_{n-1}$. In the other cases we have uniqueness; the notation is just $\tilde{M}$. These diagrams are well known; the standard reference is [Bourb 1968]. Successful presentations of sporadic groups, such as those in [CNS 1988], have been found by observing that the group in question has a subgroup which is the quotient $(\mathbb{Z} /(2))^{n-1} \cdot W(M)$ or $(\mathbb{Z} /(3))^{n-1} \cdot W(M)$ of an affine Weyl group $\mathbb{Z}^{n-1} \cdot W(M)$.

There are various definitions of hyperbolic Coxeter groups, e.g. [Bourb 1968] and [Kac 1985]. They are associated with a Reflection Representation whose bilinear form $B$ has signature $n-1,1$, but also with diagrams $M$ such that, for any proper subset $J$ of $I$, the subgroup $W_{J}$ is finite.

6.4 Further Structure Results The automorphism group of $W(M)$ has been studied only in particular cases. In [Tits 1988], it is shown that if $m_{i, j} \in\{1,2, \infty\}$ for all $i, j \in I$ and $(I, M)$ has no triangles, then Aut $W$ is the semi-direct product of $W$ and a finite group that can be determined directly from $M$. See [Howl 1988] for a way to read off the Schur index from $M$ (as well as the proof that the multiplier itself is an elementary abelian 2-group).

In [delaH 1987] it is shown that if $W$ is infinite, then either $W$ contains a free nonabelian subgroup or $W$ has an abelian subgroup of finite index. (Moreover, if the former case does not hold, then $B_{M}$ is degenerate).

For descriptions of involutions in $W$, see [Deo 1982] and [Spri 1982].

6.5 Problem Suppose $(I, M)$ and $\left(I^{\prime}, M^{\prime}\right)$ are nonisomorphic Coxeter diagrams (with $I$ and $I^{\prime}$ finite). Is it true that $W(M) \neq W\left(M^{\prime}\right)$ if $M$ and $M^{\prime}$ are connected? (A counterexample to the non-connected case is furnished by the dihedral group of order 12 which is isomorphic to both $W\left(I_{2}^{6}\right)$ and $W\left(I_{2}^{2} \cup I_{2}^{3}\right)$.)

6.6 Related concepts If the conditions $r^{2}=1$ are removed from the Coxeter group presentation, the Braid group presentation results. They arise as monodromy groups of the complement in the complexified Reflection Representation space of the union of the reflection nyperplanes (cf. [Hend 1985]).

Let $(W, R)$ be a Coxeter system of type $M$. The Coxeter monoid of type $M$ has presentation

$$
r s r \cdots\left[m_{r s} \text { factors }\right]=s r s \cdots\left[m_{r s} \text { factors }\right] \text { if } m_{r s}<\infty \text {, and } r r=r \text { for all } r, s \in R \text {. }
$$

Part (ii) of the proof of 5.1 deals with a quotient of such a monoid. The set of all so-called Demazure operators on the character ring of a fixed maximal torus of semi-simple Lie group form a monoid of this kind (these are the $\sigma_{i}$ on p. 411 of [Jantz 1987], see also [Jos 1985]). Recent work [RiSp 1989] on orbits in the flag variety $G / B$ (over an algebraically closed field 
of characteristic $\neq 2$; see $\S 7$ ) of a $\sigma$-fixed point subgroup of $G$, where $\sigma$ is an involutory automorphism of $G$, also makes use of this Coxeter monoid.

In [Tsar 1990], finiteness questions concerning these monoids and generalizations are studied; this has an application to incidence geometry and group amalgams in that it provides a sufficient condition for finiteness of the locally finite geometry/group. To be more specific, suppose $G$ is a group generated by a collection of subgroups $\left\{G_{r}\right\}_{r \in R}$ with the property that $\left\langle G_{r}, G_{s}\right\rangle$ is finite for all $r, s \in R$. Then there exists a Coxeter matrix $M=\left(m_{i j}\right)$ with $\left\langle G_{r}, G_{s}\right\rangle=G_{r} G_{s} G_{r} \cdots\left[m_{r s}<\infty\right.$ factors $]=G_{s} G_{r} G_{s} \cdots\left[m_{r s}<\infty\right.$ factors $]$. If the Coxeter monoid of type $M$ is finite (or contains an element $w_{0}$ with $r w_{0}=w_{0} r=w_{0}$ for all $r \in R$ ), the group $G$ is finite.

\section{Geometry}

A fundamental approach to the geometry underlying groups of Lie type has been given by Tits in his theory of buildings. The origins are to be found in Tits' book [Tits 1974] and his paper [Tits 1981], introductions into the subject matter in [Brown 1989] and [Ron 1989]. Here we follow [BuCo 1990] and treat part of the axiomatic theory in terms of chamber systems. Starting point is the notion of generalized polygon.

A chamber system over $I$ is a set $\mathcal{C}$ (whose members are called chambers) together with a set of equivalence relations $\underset{i}{\sim}$, one for each $i \in I$. The equivalence classes are called panels. More generally, connected components of $\left(\mathcal{C}, \bigcup_{j \in J} \sim_{j}\right)$ for a subset $J$ of $I$ are called $J$-cells. The example to bear in mind is $\mathcal{C}\left(G / B ;\left\{P_{i}\right\}_{i \in I}\right)$, where $G$ is a group and $\left\{P_{i}\right\}_{i \in I}$ is a collection of subgroups of $G$ containing the subgroup $B$; here the chambers are the cosets with respect to $B$ and the relation $x B \underset{i}{\sim} y B$ stands for $y^{-1} x \in P_{i}$. The $J$-cells correspond to the elements of $G /\left\langle P_{i} \mid i \in J\right\rangle$. The chamber system $\mathcal{C}(W, R)$ is of this shape: it coincides with $\mathcal{C}\left(W / 1 ;\{\langle r\rangle\}_{r \in R}\right)$. Each $J$-cell is a chamber systems over $J$ in its own right. We usually assume that $\mathcal{C}$ is connected, that is, $\mathcal{C}$ constitutes a single $I$-cell. For $\mathcal{C}\left(G / B ;\left\{P_{i}\right\}_{i \in I}\right)$ as above this means that we assume $G=\left\langle P_{i} \mid i \in I\right\rangle$. The relation $\sim$ stands for the union of $\sim$ over all $j \in I$. A type of a path $c_{0}, c_{1}, \ldots, c_{q}$ in the graph $(\mathcal{C}, \sim)$ (of length $q$ ) is a word $i_{1}^{j} \cdots i_{q} \in I^{*}$ such that $c_{j-1} \underset{i_{j}}{\sim} c_{j}$ for each $j \in\{1, \ldots, q\}$. In general the type of a path need not be unique, but it will turn out be in the examples we are interested in. We shall say that a word in $I^{*}$ is simple if it never contains the same letter (from $I$ ) twice in a row.

7.1 Generalized polygons A chamber system $\mathcal{C}$ over an index set $I$ of size two is called a generalized $m$-gon (cf. [Tits 1959]) if

(i) each equivalence class has at least 2 chambers;

(ii) the diameter of the graph $(\mathcal{C}, \sim)$ is $m$; moreover, for each panel $p$ and chamber $c$ there is a path of length at most $m-1$ in $(\mathcal{C}, \sim)$ connecting $c$ to a chamber in $p$;

(iii) the graph $(\mathcal{C}, \sim)$ contains no closed simple paths of length less than $2 \mathrm{~m}$.

For $m>1$, condition (iii) implies that the type of a path is indeed unique. In the finite case, the size of the $j$-cells only depends on the type $j \in I$. These integers $s$ and $t$ are known as the parameters of the generalized $n$-gon $\mathcal{C}$. If $s, t>1$, the only $m>1$ for which generalized $m$-gons may be found to occur are $2,3,4,6,8$. Also, if $m$ is odd then $s=t$ (cf. [BCN 1968] for references).

For motivation, first analyze the case where $s=t=1$. Then the graph $(\mathcal{C}, \sim)$ is 
connected of valency 2 and has diameter $n$, so consists of a single circuit of length $2 n$ with labels alternating between the two letters from $I$. In other words, $\mathcal{C}=\mathcal{C}(W, R)$ (cf. $\S 2$ ), where $(W, R)$ is the Coxeter system of type $I_{2}^{m}$.

Next consider the case of $m=3$. Set $I=\{i, j\}$. Letting $P$ and $L$, respectively, be the collections of $i$-cells and of $j$-cells, respectively, and defining incidence $\mathcal{I}$ to be the subset of $P \times L$ consisting of all $(p, l)$ such that $p \cap l \neq \emptyset$, we obtain a projective plane $(P, L, \mathcal{I})$. Conversely, a generalized 3 -gon results from any projective plane $(P, L, I)$ by letting the $\mathcal{C}$ be the set of all pairs $(p, l) \in \mathcal{I}$ consisting of a point $p \in P$ and a line $l \in L$ incident to it, and by letting $\underset{i}{\sim}$ and $\underset{j}{\sim}$ stand for having a point, respectively, line in common.

In a similar way, generalized 4-gons can be identified with incidence systems in which every point has a unique point collinear to it on any line not containing it (a so-called polar space of rank 2).

Consider again the general case of a generalized $m$-gon $\mathcal{C}(m>1)$. Write $I=\{i, j\}$. If $c, d$ are chambers at distance $<m$, there is a unique simple path from $c$ to $d$ and so we can attach a unique type to the ordered pair $(c, d)$. The only remaining case is when $c, d$ are at distance $m$. Then the type of a simple path $c \sim c_{1} \cdots \sim d$ is $i j i \cdots$ or $j i j \cdots$ according as $c \underset{i}{\sim} c_{1}$ or $\underset{j}{\sim} \sim_{1}$. On the other hand, in view of (ii) both panels $c i^{*}$ (notation for the $\{i\}$-cell containing $c$ ) and $c j^{*}$ must contain a chamber at distance $m-1$, leading to paths of either type. Thus, letting $F$ be the free monoid on $I$ subject to the relations

$$
i j i \cdots[m \text { factors }]=j i j \cdots[m \text { factors }] \text { if } m<\infty,
$$

we obtain a map $\mathcal{C} \times \mathcal{C} \rightarrow F$ sending $(c, d)$ to the type of a simple path of minimal length from $c$ to $d$.

7.2 Chamber systems of type $M$ If $(I, M)$ is a Coxeter diagram, the chamber system is said to be of type $M$ if, for any distinct $i, j \in I$, every $\{i, j\}$-cell is a generalized $m_{i, j}$-gon.

7.3 Lemma Let $c, d$ be chambers of a chamber system $\mathcal{C}$ of type $M$. Then

(i) the type of a minimal path from $c$ to $d$ is a minimal expression in $I^{*}$;

(ii) if $\mathbf{i} \in I^{*}$ is a minimal expression and if $\mathbf{i}$ is the type of a path from $c$ to $d$, then, for each $\mathbf{i}^{\prime} \in I^{*}$ with $\rho\left(\mathbf{i}^{\prime}\right)=\rho(\mathbf{i})$, there is a path from $c$ to $d$.

Proof (i) Suppose $c=c_{0} \sim c_{1} \sim \ldots \sim c_{q}=d$ is a path from $c$ to $d$ with type i. If $\mathbf{i}=i j i \ldots$ ( $m_{i, j}$ factors), then, by the axiom for chamber systems of type $M$, there is another path from $c$ to $d$ with type $j i j \cdots$ ( $m_{i, j}$ factors). Now, applying this observation to subpaths as well, we obtain from Theorem 5.3 that, if $\mathbf{i}$ is not minimal, we may assume without loss of generality that, for some $j \in\{1, \ldots, q-1\}$ and $k \in I$, the subpath $c_{j-1} \sim c_{j} \sim c_{j+1}$ has type $k k$. But then $c=c_{0} \sim c_{1} \sim \ldots \sim c_{j-1} \sim c_{j+1} \sim c_{q}=d$ is a path from $c$ to $d$ which is strictly shorter than the one we started with. Hence (i).

(ii) In view of Theorem 5.3, (i), and induction on the length of $i^{\prime}$, it suffices to show that if $i_{1} i_{2} \cdots i_{q}$ is the type of a path from $c$ to $d$, then for each $j(1 \leq j<q)$ and each $k \in I$, there is a path from $c$ to $d$ of type $i_{1} i_{2} \cdots i_{j} k k i_{j+1} \cdots i_{q}$. But this is direct from the definition of chamber system.

QED

The lemma shows that, given two chambers $c$ and $d$, there is an element $w \in W$ such that for each word $\mathbf{i} \in \rho^{-1}(w)$ we can find a path from $c$ to $d$ of type $\mathrm{i}$. For thin chamber systems, 
the universal object $\mathcal{C}(W, R)$ differs from its quotients in that the element $w$ is uniquely determined by $c$ and $d$. The following definition is inspired by this observation. If the type i of a path is a minimal expression for $\rho(\mathbf{i})$, we also say, par abus de language, that the type is minimal.

7.4 Definition A connected chamber system of type $M$ over $R$ is called a building of type $M$ if every simple closed path with minimal type is trivial (i.e., consists of a single chamber).

7.5 Examples (i) For any Coxeter system $(W, R)$ of type $M$, the chamber system $\mathcal{C}(W, R)$ is a building of type $M$. All panels have size 2 . It is referred to as an aptment.

(ii) Take $\mathcal{C}$ to be the set of maximal flags of a projective geometry of rank $n$, and if $i \in$ $\{0, \ldots, n-1\}$, set $\underset{i}{\sim}$ for the relation 'coinciding in all subspaces except possibly the $i$-th'. Then $\mathcal{C}$ is a building of type $A_{n}$. For, first of all, $\mathcal{C}$ has type $A_{n}$ by what we have seen in $\S 7.1$. Second, suppose $\gamma$ is a closed simple path of $\mathcal{C}$ with minimal type $i \in I^{*}$ and origin $c$. In view of induction on $n$, we may assume $i_{1} \in I_{\rho(\mathrm{i})}$. Then according to 3.1 (iii), we can write $\rho(\mathrm{i})=$ $w_{1} r_{1} w_{2}$ with $w_{1}, w_{2} \in\left\langle R \backslash\left\{r_{1}\right\}\right\rangle$, where $r_{1}=\rho(1)$ belongs to the first node of $A_{n}$. Moreover, by 5.1 .(iv), we can choose $w_{1}, w_{2} \in\left\langle R \backslash\left\{r_{1}\right\}\right\rangle$ in such a way that $l(\mathrm{i})=l\left(w_{1}\right)+1+l\left(w_{2}\right)$, so that, by the previous lemma, we may assume without loss of generality that $\gamma$ has type $\left(\mathbf{i}^{\prime}, 1, \mathbf{i}^{\prime \prime}\right)$ with $\mathbf{i}^{\prime}, \mathbf{i}^{\prime \prime} \in(I \backslash\{1\})^{*}$. Let $d, e$ be the 1-adjacent chambers of $\gamma$ (occurring in this order). Then $d, e \in c(I \backslash\{1\})^{*}$ so $d \in e(I \backslash\{1\})^{*} \cap e\{1\}^{*}=\{e\}$, contradicting the simplicity of $\gamma$.

(iii) Let $P$ be a set of 7 points. There are 30 choices $L$ of collections 7 subsets of size three such that $(P, L, \mathcal{I})$, where $\mathcal{I} \subset P \times L$ is the usual inclusion relation, is a projective plane - a so-called Fano plane. The group Alt ${ }_{7}$ has two orbits on the family of 30 planes, each of size 15. Let $\Pi$ be one such orbit. We construct a chamber system $C$ of type $B_{3}$ as follows: the chambers are the triples $(p, l, \pi)$ with $p \in P$ and $l$ a line of the plane $\pi \in \Pi$ containing $p$. If $i=1,2,3$, two triples are called $i$-adjacent if they agree in all entries $\neq i$. The resulting chamber system is not a building of type $B_{3}$. This follows from the existence of a closed path of type 123123123.

(iv) Starting with a polytope $\Pi$ in $\mathbb{R}^{n}$, one obtains a chamber system $\mathcal{C}(\Pi)$ over $I=$ $\{0, \ldots, n-1\}$ by taking as chambers the maximal simplices (consisting of a vertex (a 0 face), an edge (a 1-face) on the vertex, a 2-face on the edge, and so on) and letting two simplices be $i$-adjacent whenever they agree in all $j$-faces for $j \neq i$. If the polytope is combinatorially regular, the chamber system is of type $M$ for some Coxeter matrix $M$. Any such chamber system can be obtained as a quotient of the chamber system $\mathcal{C}(W, R)$ where $(W, R)$ is the Coxeter system of type $M$. Using the topology induced from $\mathbb{R}^{n}$, it can then be shown that $\mathcal{C}(\Pi) \cong \mathcal{C}(W, R)$ (result of [McMu 1967], cf. [DrSc 1988]).

(v) If $\mathcal{C}^{\prime}$ is a building of type $M^{\prime}$, where $M^{\prime}$ is a Coxeter matrix over $R^{\prime}$, then the direct product $\mathcal{C} \times \mathcal{C}^{\prime}$ is a building of type $M \cup M^{\prime}$ over $R \cup R^{\prime}$.

(vi) Let $(P, \mathcal{L})$ be a polar space, that is, for each pair $p, l \in P \times \mathcal{L}$, the set of points in $l$ collinear with $p$ is either a singleton or $l$. Motivating examples are obtained by taking the absolute points and lines of a polarity in projective space. Assume further that $(P, \mathcal{L})$ is nondegenerate: no point is collinear with each point in $P$. A singular subspace is a subset $X$ of $P$ with the property that any two of its points are collinear and that all points on a line 
joining them belong to $X$. Then maximal singular subspaces exist and have the structure of projective spaces. Assume further that all lines have at least three points and that the maximal rank of a maximal singular subspace is $n-1<\infty$. Then a chamber system of type $B_{n}$ can be constructed whose chambers are the maximal chains of singular subspaces (with respect to inclusion) and in which two chambers are $i$-adjacent if and only if their members (singular subspaces) coincide in all but (possibly) the $i$-th member (counting 1 for points, 2 for lines, and $n$ for maximal singular subspaces). The chamber system thus constructed is a building. Conversely, the 1- and 2-cells of any building of type $B_{n}$ form a non- degenerate polar space of rank $n$. See [Tits 1974]; an elementary treatment will appear in [BuCo 1990].

7.6 Proposition Suppose $\mathcal{C}$ is a building of type $M$. Then

(i) For every pair $\mathbf{i}, \mathbf{i}^{\prime} \in R^{*}$ of minimal expressions, the existence of two simple paths with common origin and common end point, and types $\mathbf{i}$ and $\mathbf{i}^{\prime}$, respectively, implies $\rho(\mathbf{i})=\rho\left(\mathbf{i}^{\prime}\right)$;

(ii) for each $J \subseteq R$ and each $c \in \mathcal{C}$, the $J$-cell $c J^{*}$ is a building of type $\left.M\right|_{J \times J}$.

Proof (i) Denote the two paths of type $\mathrm{i}, \mathrm{i}^{\prime}$ by $\gamma, \gamma^{\prime}$, respectively, and let $c, d$ be their common origin and end point, respectively. We proceed by induction on $l(\mathbf{i})$. If $l(\mathbf{i})=0$, then $c=d$ (and $\rho(\mathbf{i})=1)$ so $\rho\left(\mathrm{i}^{\prime}\right)=1$ by the definition of chamber system, whence $\rho(\mathrm{i})=\rho\left(\mathrm{i}^{\prime}\right)$ as required. Assume $l(\mathrm{i})>0$. Then there is $j \in I$ with $l(\rho(j \mathrm{i}))<l(\mathrm{i})$. In view of 4.1 (vii) and 7.4(ii), we may assume that the first element of $i$ is $j$. Thus, $i=j i^{\prime \prime}$ for some $i^{\prime \prime} \in I^{*}$. Let $e$ be the chamber of $\gamma$ following $c$ and denote by $\gamma^{\prime \prime}$ the tail of $\gamma$ starting at $e$. Clearly, $\gamma^{\prime \prime}$ is a simple path from $e$ to $d$ with minimal type $\mathbf{i}^{\prime \prime}$ and of length $l(\mathbf{i})-1$. If $l\left(\rho\left(j \mathbf{i}^{\prime}\right)\right)>l\left(\mathbf{i}^{\prime}\right)$, then $\left(e, \gamma^{\prime}\right)$ is a simple path from $e$ to $d$ of minimal type $j \mathrm{i}^{\prime}$, so by the induction hypothesis applied to $\gamma^{\prime \prime}$ and $\left(e, \gamma^{\prime}\right)$, we have $\rho\left(\mathbf{i}^{\prime \prime}\right)=\rho\left(j \mathbf{i}^{\prime}\right)$, leading to $\rho(\mathbf{i})=\rho\left(\mathbf{i}^{\prime}\right)$ as desired.

Suppose, therefore, that $l\left(\rho_{j} \rho\left(\mathbf{i}^{\prime}\right)\right)<l\left(\mathbf{i}^{\prime}\right)$. Then, as before, we may assume, without loss of generality, that $\mathbf{i}^{\prime}=j \dot{i}^{\prime \prime \prime}$ where $\dot{i}^{\prime \prime \prime}$ is minimal. Denote by $e^{\prime}$ the chamber of $\gamma^{\prime}$ following $c$ and by $\gamma^{\prime \prime \prime}$ the tail of $\gamma^{\prime}$ with origin $e^{\prime}$. If $e \neq e^{\prime}$, then $\left(e, \gamma^{\prime \prime \prime}\right)$ is a simple path from $e$ to $d$ with minimal type $j \mathbf{i}^{\prime \prime \prime}$, so by the induction hypothesis $\rho\left(\mathbf{i}^{\prime \prime}\right)=\rho_{j} \rho\left(\mathbf{i}^{\prime \prime \prime}\right)$, whence $j \mathbf{i}^{\prime \prime \prime}$ is a minimal expression for $\mathrm{i}^{\prime \prime}$, contradicting $l\left(j{ }^{\prime \prime}\right)=l(\mathbf{i})>l\left(\rho_{j} \rho(\mathbf{i})\right)$. Hence, $e=e^{\prime}$ and, again by the induction hypothesis, $\rho\left(\mathrm{i}^{\prime \prime}\right)=\rho\left(\mathbf{i}^{\prime \prime \prime}\right)$, so that $\rho(\mathbf{i})=\rho\left(j \mathbf{i}^{\prime \prime}\right)=\rho\left(j \mathbf{i}^{\prime \prime \prime}\right)=\rho\left(\mathbf{i}^{\prime}\right)$. This establishes (i).

(ii) is immediate from the definition of chamber system.

The axiom of buildings can be weakened. This is the context of the theorem below (cf. [Tits 1981]). As a consequence of its proof, we obtain some elementary properties of buildings.

7.7 Theorem Let $\mathcal{C}$ be a connected chamber system of type $M$ over $I$. Suppose there is a chamber $c$ such that for every pair $\mathbf{i}, \mathrm{i}^{\prime} \in I^{*}$ of minimal expressions, the existence of simple paths with common origin $c$ and common endpoint, and types $\mathbf{i}, \mathbf{i}^{\prime}$, respectively, implies $\rho(\mathrm{i})=\rho\left(\mathrm{i}^{\prime}\right)$. Then $\mathcal{C}$ is a building.

Proof Let $d$ be a chamber, and let $\gamma, \gamma^{\prime}$ be two distinct paths starting at $d$, with the same endpoint, and with respective minimal types $\mathbf{i}$ and $\mathbf{i}^{\prime}$. We show that $\rho(\mathbf{i})=\rho\left(\mathbf{i}^{\prime}\right)$. Note that this suffices for the proof that $\mathcal{C}$ is obviously a building. 
In view of induction on the length of a path from $c$ to $d$ (and connectedness of $\mathcal{C}$ ) it suffices to establish $\rho(\mathbf{i})=\rho\left(\mathbf{i}^{\prime}\right)$ in the case where $c \sim d$. Thus, suppose $c \in d\left(j^{*}\right)$ for some $j \in I$. The paths $(c, \gamma)$ and $\left(c, \gamma^{\prime}\right)$ have types $j i$, and $j \mathbf{i}^{\prime}$, respectively. If these types are both minimal, then $\rho(j \mathbf{i})=\rho\left(j \mathbf{i}^{\prime}\right)$ by the hypothesis, whence $\rho_{j} \rho(\mathbf{i})=\rho_{j} \rho\left(\mathbf{i}^{\prime}\right)$, and $\rho(\mathbf{i})=\rho\left(\mathbf{i}^{\prime}\right)$, as required.

Assume that neither $j \mathbf{i}$ nor $j \mathbf{i}^{\prime}$ is minimal. Then, by 4.1 (vii) there are minimal expressions $j \mathbf{i}^{\prime \prime}, j \mathbf{i}^{\prime \prime \prime} \in I^{*}$ such that $\rho(\mathbf{i})=\rho\left(j \mathbf{i}^{\prime \prime}\right)$ and $\rho\left(\mathbf{i}^{\prime}\right)=\rho\left(j \mathbf{i}^{\prime \prime \prime}\right)$. According to Lemma 7.4 there are minimal paths with the same origin and endpoint as $\gamma\left(\right.$ and $\gamma^{\prime}$ ) with types $j \mathbf{i}^{\prime \prime}$ and $j \mathbf{i}^{\prime \prime \prime}$, respectively. Let the first point following $d$ on these paths be $e, e^{\prime}$, respectively. Denote the tail of these paths from $e, e^{\prime}$ respectively (to the end), by $\gamma^{\prime \prime}, \gamma^{\prime \prime \prime}$, respectively.

If $e^{\prime}=c \neq e$, then the paths $\left(c, \gamma^{\prime \prime}\right)$ and $\gamma^{\prime \prime \prime}$ both start at $c$ and have types $j \mathbf{i}^{\prime \prime}$ and $\mathbf{i}^{\prime \prime \prime}$, respectively; both are minimal and have the same extremities, so $\rho\left(j \dot{i}^{\prime \prime}\right)=\rho\left(\mathrm{i}^{\prime \prime \prime}\right)$. Hence, $l\left(\mathbf{i}^{\prime \prime}\right)+1=l\left(j \mathbf{i}^{\prime \prime}\right)=l\left(\mathbf{i}^{\prime \prime \prime}\right)$ in view of minimality. But then also $\rho\left(\mathbf{i}^{\prime \prime}\right)=\rho\left(j \mathbf{i}^{\prime \prime \prime}\right)$, whence $l\left(\mathbf{i}^{\prime \prime}\right)=l\left(j \mathbf{i}^{\prime \prime \prime}\right)=l\left(\mathbf{i}^{\prime \prime \prime}\right)+1$, a contradiction with minimality. Similarly, we can rule out $e=c \neq e^{\prime}$. If $c \neq e, e^{\prime}$, then $\left(c, \gamma^{\prime \prime}\right)$ and $\left(c, \gamma^{\prime \prime \prime}\right)$ are paths of type $j \mathrm{i}^{\prime \prime}$ and $j \mathrm{i}^{\prime \prime \prime}$, respectively, with the same extremities, and if $c=e=e^{\prime}$, then $\gamma^{\prime \prime}$ and $\gamma^{\prime \prime \prime}$ are paths of type $\mathbf{i}^{\prime \prime}$ and $\mathbf{i}^{\prime \prime \prime}$, respectively. In both cases the required conclusion follows from the hypotheses.

Finally, assume $j \mathbf{i}^{\prime}$ is minimal, but $j \mathbf{i}$ is not (the other remaining case being the same up to a change of rôles for $\mathbf{i}$ and $\left.\mathbf{i}^{\prime}\right)$. Again, replace $\mathbf{i}$ by $j \ddot{i}^{\prime \prime}$ such that $\rho(\mathbf{i})=\rho\left(j \mathbf{i}^{\prime \prime}\right)$, denote by $e$ the first member following $d$ on a simple path with same extremities as $\gamma$ and type $j \mathrm{i}^{\prime \prime}$, and by $\gamma^{\prime \prime}$ the tail end of this path starting at $e$. If $c=e$, then $c, \gamma^{\prime}$ and $\gamma^{\prime \prime}$ are paths starting at $c$ with the same endpoints, having types $j \mathrm{i}^{\prime}$ and $\mathrm{i}^{\prime \prime}$, respectively. Thus, by the hypothesis, $\rho\left(j \mathbf{i}^{\prime}\right)=\rho\left(\mathbf{i}^{\prime \prime}\right)$, whence $\rho\left(\mathbf{i}^{\prime}\right)=\rho\left(j \mathbf{i}^{\prime \prime}\right)=\rho(\mathbf{i})$, as required. Therefore, we may restrict attention to the case where $c \neq e$. Consideration of the paths $c, \gamma^{\prime}$ and $c, e, \gamma^{\prime \prime}$ leads to $\rho\left(j \mathbf{i}^{\prime}\right)=\rho\left(j \mathbf{i}^{\prime \prime}\right)$, whence $\rho\left(\mathbf{i}^{\prime}\right)=\rho\left(\mathbf{i}^{\prime \prime}\right)$. Upon replacing $\gamma^{\prime}$ by a suitable path, we may assume $\mathbf{i}^{\prime \prime}=\mathbf{i}^{\prime}$. Now $c, \gamma^{\prime}$ and $c, e, \gamma^{\prime \prime}$ are paths starting at $c$ with the same endpoint and the same type $j \mathrm{i}^{\prime}$. Since $d \neq e$ (due to the simplicity of $d, \gamma^{\prime \prime}$ ), the following assertion shows that we have a contradiction, thus finishing the proof of the theorem.

$\left(^{*}\right)$ If two simple paths starting at $c$ have the same extremities and the same minimal type, they coincide.

It remains to establish $\left(^{*}\right)$. Let $\delta, \delta^{\prime}$ be two simple paths, from $c$ to $b$ with minimal type i. We proceed by induction on $l(\mathbf{i})$. The case $l(\mathbf{i})=0$ being trivial, assume $l(\mathbf{i})>0$. Thus $\delta$ has tail $h, b$ and $\delta^{\prime}$ has tail $h^{\prime}, b$ for chambers $h, h^{\prime}$ which are $j$-adjacent to $e$ for some $j \in I$. Let $\delta^{\prime \prime}, \delta^{\prime \prime \prime}$ be the head part of $\delta, \delta^{\prime}$ ending at $h, h^{\prime}$, respectively. These paths have minimal type $\mathbf{i}^{\prime}$ such that $\mathbf{i}=\mathbf{i}^{\prime} j$. If $h \neq h^{\prime}$, then $\delta^{\prime \prime}$ and $\left(\delta^{\prime \prime \prime}, h\right)$ are paths starting at $c$ and ending at $h$ with minimal types $\mathrm{i}^{\prime}$ and $\mathrm{i}$, respectively. According to the hypothesis, this implies $\rho\left(\mathbf{i}^{\prime}\right)=\rho(\mathbf{i})$. This is absurd as $\rho(\mathbf{i})=\rho\left(\mathbf{i}^{\prime}\right) \rho_{j}$. Therefore, $h=h^{\prime}$, and we can finish by invoking the induction hypothesis.

QED

7.8 Corollary Suppose $\mathcal{C}$ is a building of type $M$, and $c, d, e$ are chambers of $\mathcal{C}$. Then

(i) there is a unique element in $W$, denoted by $\rho(c, d)$, such that the type map induces a bijective correspondence

typ : $\{$ minimal paths from $c$ to $d\} \rightarrow I_{\rho(c, d)}^{*}$; 
(ii) if $\gamma$ is a simple path from $c$ to $d$ with minimal type, then $\gamma$ is minimal, and $\rho(c, d)=$ $\rho(\operatorname{typ}(\gamma))$

(iii) if $c \in d j^{*}$, where $j \in I$, then $\rho(c, e) \in\left\langle\rho_{j}\right\rangle \rho(d, e)$;

(iv) the map $x \mapsto \rho(d, x)$ is a morphism $\mathcal{C} \rightarrow \mathcal{C}(W, R)$ of chamber systems over $I$;

(v) $l(\rho(c, d) \rho(d, e)) \leq l(\rho(c, e))$.

Proof (i) Let $\gamma, \gamma^{\prime}$ be minimal paths from $c$ to $d$. Then $\operatorname{typ}(\gamma)$ and $\operatorname{typ}\left(\gamma^{\prime}\right)$ are minimal by part (i) of the lemma, so by the definition of building, there is $w \in W$ with $\operatorname{typ}(\gamma), \operatorname{typ}\left(\gamma^{\prime}\right) \in$ $I_{w}^{*}$. If $t y p \gamma=t y p \gamma^{\prime}$, then $\gamma=\gamma^{\prime}$ by $\left(^{*}\right)$ of the proof above. Finally, typ is surjective onto $I_{w}^{*}$ by part (ii) of the lemma.

(ii) This is immediate from (i) and the definition of a building.

(iii) Let $\gamma$ be a minimal path from $d$ to $e$ with type, say, i. Thus $\rho(\mathrm{i})=\rho(d, e)$. Consider the path $(c, \gamma)$. If $c=d$, there is nothing to show, so we may assume this path is simple. If $j \mathrm{i}$ is a minimal expression, then, by (ii), the path $(c, \gamma)$ is minimal, and $\rho(c, e)=\rho_{j} \rho(\mathbf{i}) \epsilon$ $\left\langle\rho_{j}\right\rangle \rho(d, e)$. Otherwise, we may assume without loss of generality (cf. 4.1(vii) and 7.4) that $\mathrm{i}=j \mathrm{i}^{\prime}$. Let $d^{\prime}$ be the first chamber of $\gamma$ following $d$. Then $d^{\prime} \in c j^{*}$, so either $d^{\prime}=c$ and there is a path from $c$ to $e$ of type $\mathbf{i}^{\prime}$, or $d^{\prime} \neq c$ and there is a path from $c$ (via $d^{\prime}$ ) to $e$ of type $\mathrm{ji}^{\prime}$. Since both types are minimal, we have, again by (ii), that

$$
\rho(c, e) \in\left\{\rho\left(\mathbf{i}^{\prime}\right), \rho\left(j \mathbf{i}^{\prime}\right)\right\}=\left\{\rho(\mathbf{i}), \rho_{j} \rho(\mathbf{i})\right\}=\left\langle\rho_{j}\right\rangle \rho(d, e) .
$$

Hence (iii).

(iv) Let $x, x^{\prime}$ be two $j$-adjacent chambers in $\mathcal{C}$, where $j \in I$. Then, by (iii), $\rho(x, d) \in$ $\left\langle\rho_{j}\right\rangle \rho\left(x^{\prime}, d\right)$. Since, obviously, $\rho(c, e)=\rho(e, c)^{-1}$, it follows that $\rho(d, x) \in \rho\left(d, x^{\prime}\right)\left\langle\rho_{j}\right\rangle$, which is equivalent to saying that $\rho(d, x)$ and $\rho\left(d, x^{\prime}\right)$ are $j$-adjacent in $\mathcal{C}(W, R)$. This establishes (iv).

(v) Since distances decrease under morphisms, it follows from (iv) that

$$
d_{\mathcal{C}(W, R)}(\rho(d, c), \rho(d, e)) \leq d(c, e)
$$

But, according to the remark at the end of $\S 2$, the left hand side is equal to $l\left(\rho(d, c)^{-1} \rho(d, e)\right)$, and, in view of (i), we have $d(c, e)=l(\rho(c, e))$. Hence the corollary.

7.9 Apartments We shall now see that the study of Coxeter chamber systems is of good use to buildings. In accordance with 7.5(i), an apartment of a chamber system $\mathcal{C}$ of type $M$ is a subsystem which is isomorphic to $\mathcal{C}(W, R)$.

Observe that if $\alpha: \mathcal{C}(W, R) \rightarrow \mathcal{C}$ is an injective morphism, then $d(\alpha(x), \alpha(y))=d(x, y)$. In order to find apartments in $\mathcal{C}$, we have to find subsets $A$ of chambers with the property that the restriction to $A$ of the morphism $x \mapsto \rho(c, x)$ is injective. In particular, for $d, e \in A$ we will have equality in part $(\mathrm{v})$ of the corollary.

7.10 Theorem Let $\mathcal{C}$ be a building.

(i) Every pair of chambers of $\mathcal{C}$ is contained in an apartment; 
(ii) if $A$ is an apartment of $\mathcal{C}$ containing the chambers $c, d$, then every minimal path from $c$ to $d$ is entirely contained in $A$.

Proof For $X \subseteq W$, a mapping $\alpha: X \rightarrow \mathcal{C}$ is called a strong isometry if $\rho(\alpha(x), \alpha(y))=x^{-1} y$ for all $x, y \in X$. The theorem will be an easy consequence of the following assertion:

(**) If $\alpha: X \rightarrow \mathcal{C}$ is a strong isometry then $\alpha$ can be extended to a strong isometry $\alpha: W \rightarrow \mathcal{C}$.

Let us first prove the assertion. By Zorn's lemma, it suffices to show that if $X \subset W$, there is $w \in W \backslash X$ such that $\alpha$ can be extended to a strong isometry on $X \cup\{w\}$. If $X=\emptyset$, we can take any $w \in W$, so assume $X \neq \emptyset$. By connectedness of $\mathcal{C}(W, R)$, there must be an $s-$ adjacent pair of chambers for some $s \in R$, such that only one of the two is in $X$. Applying an automorphism on $\mathcal{C}(W, R)$ if necessary, we may assume this pair to be $\{1, s\}$ so that $1 \in X$, and $s \notin X$. If $l(s x)>l(x)$ for all $x \in X$, then, letting $\alpha(s)$ be any chamber $s$-adjacent to $\alpha(1)$, every minimal path $\gamma$ on $\alpha(1)$ to $\alpha(x)$ extends to a minimal path $(\alpha(s), \gamma)$ from $\alpha(s)$ to $\alpha(x)$, so that $\rho(\alpha(s), \alpha(x))=s x$ for all $x \in X$. This shows that $\alpha$ extends to a strong isometry on $X \cup\{s\}$.

Therefore, assume $l(s y)<l(y)$ for some $y \in X$. Let $\alpha(s)$ be the second chamber of a minimal path from $\alpha(1)$ to $\alpha(y)$ whose type begins with $s$ (such a path exists by familiar arguments). Now $\rho(\alpha(s), \alpha(y))=s y$ by construction of $\alpha(s)$. We have to show that $\rho(\alpha(s), \alpha(x))=s x$ for all $x \in X$. Take $x \in X$. Since $\rho(\alpha(1), \alpha(x))=x$ by the hypothesis on $\alpha$, Corollary 7.8 yields $\rho(\alpha(s), \alpha(x)) \in\langle s\rangle x$. Suppose $\rho(\alpha(s), \alpha(x))=x$. Then, clearly, $l(s x)<l(x)$. Moreover, by Corollary 7.8, $l\left(\rho(\alpha(x), \alpha(s)) \rho(\alpha(s), \alpha(y)) \leq l(\rho(\alpha(x), \alpha(y)))\right.$, whence $l\left(x^{-1} s y\right) \leq l\left(x^{-1} y\right)$. This contradicts Theorem 5.2(vii). Therefore $\rho(\alpha(1), \alpha(x)) \in\langle s\rangle x \backslash\{x\}=\{s x\}$, and $\rho(\alpha(1), \alpha(x))=s x$ as required for $\alpha$ to be a strong isometry on $X \cup\{s\}$. This establishes $(* *)$.

(i) follows from (**) by taking $X=\{1, \rho(c, d)\}, \alpha(1)=c$, and $\alpha(\rho(c, d))=d$ for a pair $\{c, d\}$ of chambers of $\mathcal{C}$.

(ii) is a direct consequence of part (i) of the corollary as $\mathcal{C}(W, R)$ contains a path from $c$ to $d$ for each minimal expression of $\rho(c, d)$.

QED

In the spherical case, two opposite chambers are in a unique apartment.

7.11 Retractions Take a building $\mathcal{C}$ of type $M$ over $R$ and let $c, A$ be a pair consisting of a chamber $c$ and an apartment $A$ containing it. For each $x \in \mathcal{C}$, there is a unique chamber in $A$, called the retract of $x$ onto $A$ with center $c$, denoted by $\rho_{c, A}(x)$, with the property that $\rho(c, x)=\rho\left(c, \rho_{c, A}(x)\right)$.

Since $A$ is an apartment, there is a strong isometry $\alpha: W \rightarrow \mathcal{C}$ with $\alpha(W)=A$ and $\alpha(1)=c$. Now $\rho_{c, A}$ is the composite of the morphism of chamber systems $x \mapsto \rho(c, x)$ (cf. Corollary 7.8(iv)) and $\alpha$, so it is a morphism onto $A$ which restricts to the identity on $A$. This justifies the name 'retract'. Observe that $\rho_{c, A}$ maps minimal paths onto minimal paths of the same type. They can be used in proving that buildings of type $M$ can be viewed as residually connected geometries of Coxeter type $M$. Example 7.5(ii) deals with a converse of this result for type $A_{n}$. In view of Example 7.5(iii) we cannot expect the converse to hold for the case $M=B_{n}$. However, a mild condition on the geometry suffices (cf. [Tits 1981]). 
Each residually connected chamber system of type $E_{6}$ is a building. Again, this is not true if $E_{6}$ is replaced by $E_{7}$.

7.12 Tits systems Let $\mathcal{C}$ be a building of type $M$ over $R$. Assuming transitivity of a group $G$ of automorphisms of $\mathcal{C}$ on the set of all pairs $(c, A)$ consisting of a chamber $c$ contained in an apartment $A$, we obtain a pair $B, N$ of subgroups of $G$ that is known as a Tits system. Thus fix $c \in \mathcal{C}$ and an apartment $A$ on $c$, and suppose that $G$ is a chamber transitive group of type preserving automorphisms of $\mathcal{C}$. Writing $B=G_{c}$, we can identify $G / B$ with the set of all chambers of $\mathcal{C}$. If $d$ is a chamber of $\mathcal{C}$, then, by Theorem 7.10 , there is an apartment containing $c$ and $d$. Therefore, assuming transitivity of $G$ on the set of all incident chamber, apartment-pairs, we obtain that $g d \in A$ for some $g \in B$; furthermore, by the same assumption, there is $n \in N_{G}(A)$ with $n(g d)=c$.

If $d$ represents the $\operatorname{coset} y B$ the latter equation is equivalent to $n g y B=B$, and hence to $y \in g^{-1} n^{-1} B$. Consequently, $G=B N_{G}(A) B$. Furthermore, Write $N=N_{G}(A)$ for the setwise stabilizer of $A$ in $G$ and $H=C_{G}(A)$ for the pointwise stabilizer. Then we have again by transitivity of $G$ and the fact that $\operatorname{Aut} C(W, R)$ coincides with $W, N / H \cong W(M)$ and $B \cap N=H$.

Putting all this together, we see that any $\operatorname{coset} g B$ can be written in the form $b w B$ where $b \in B$ and $w=n H$ represents an element of $W=N / H$. Thus, the chambers $r$-adjacent to $B$ are of the form $\operatorname{br} B(b \in B)$.

It follows from transitivity of $G_{c}$ on $c r^{*} \backslash\{c\}$ that $G_{c r^{*}}=B \cup B r B$. In particular, $r \notin$ $B, B r^{-1} B=B r B$ and $B r B r B \subseteq B \cup B r B$. Writing $P_{r}=G_{c r^{*}}$, we obtain that $\mathcal{C} \cong$ $\mathcal{C}\left(G / B ;\left\{P_{i} \mid i \in I\right\}\right)$. Thus if $d=y B$ is the endpoint of a minimal path of type $r_{1}, \ldots, r_{t}$ starting at $c$, this path may be described as follows

$$
c=B \underset{r_{1}}{\sim} b_{1} r_{1} B \underset{r_{2}}{\sim} b_{1} r_{1} b_{2} r_{2} B \sim \ldots \underset{r_{t}}{\sim} b_{1} r_{1} b_{2} r_{2} \ldots b_{t} r_{t} B=d
$$

so that $y \in b_{1} r_{1} b_{2} r_{2} \cdots b_{t} r_{t} B$. On the other hand, by what we have seen above, there are $b \in B, w \in W$ such that $y \in b w B$. But, then $r_{1} \cdots r_{t}=\rho(c, d)=\rho(B, b w B)=w$.

We have seen that, whenever $r_{1} \cdots r_{t}\left(r_{i} \in R\right)$ is a minimal expression for $w \in W$, then $B r_{1} B r_{2} \cdots B r_{t} B=B w B$. Summarizing, we have:

7.13 Proposition Let $C$ be a thick building admitting a group $G$ of automorphisms. If $G$ is transitive on the set of all pairs $\left(c^{\prime}, A^{\prime}\right)$ with $c^{\prime} \in \mathcal{C}$ and $A^{\prime}$ an apartment containing $c^{\prime}$, then the following hold where $B=G_{c}, N=N_{G}(A), H=C_{G}(A)$.

(i) $B$ and $N$ are subgroups of $G$ generating the full group $G$;

(ii) $H=B \cap N$ is a normal subgroup of $N$ and $W=N / H$;

(iii) $R$ is a generating set of $W$ satisfying the inclusion $r B w B \subseteq B w B \cup B r w B$ for any $w \in W, r \in R$

(iv) for each $r \in R$, we have $r B r^{-1} \nsubseteq B$.

Proof (i) This follows from the relation $G=B W B$.

(ii) is obvious from the above. 
(iii) If $l(r w)=l(w)+1$, this follows from the above. Otherwise, there is a minimal expression $w=r r_{2} \cdots r_{t}$ so that (as $B r B r B \subseteq B \cup B r B$ )

$$
\begin{aligned}
B r B w B & \subseteq B r B r r_{2} \cdots r_{t} B=(B r B r B)\left(B r_{2} B \cdots B r_{t} B\right) \\
& \subseteq(B \cup B r B)\left(B r_{2} \cdots r_{t} B\right)=B r w B \cup B r B r_{2} \cdots r_{t} B=B r w B \cup B w B .
\end{aligned}
$$

(iv) If $B r B r^{-1} \subseteq B$, then $B r B r B=B r B r^{-1} B=B$. But this means that $c r^{*}$, where $c=B$, consists of $c$ and $c r$ only; therefore $\mathcal{C}$ is thin.

QED

The above properties of a group $G$ are now abstracted from the chamber system setting.

7.14 Definition Let $G$ be a group. A Tits system in $G$ is a quadruple $(B, N, W, R)$ for which the conditions (i)-(iv) of the above proposition hold. (There is no requirement that $(W, R)$ be a Coxeter system - it will follow.) Thus, the content of the above proposition is that a building over $R$ supplied with a group of automorphisms gives rise to a Tits system provided that it is transitive on the set of all incident chamber, apartment pairs. There is a converse.

7.15 Theorem Let $(B, N, W, R)$ be a Tits system in the group $G$. Then

(i) the pair $(W, R)$ is a Coxeter system; for each $r \in R$ and $w \in W$, we have $l(r w)>l(w)$ if and only if $B r B w B=B r w B$;

(ii) if $J, K \subseteq R$, then, for all $r \in R$ and $w \in W$, we have $B r B w B=B r w B$ if and only if $l(r w)=l(w)+1$. Moreover, $B\langle J\rangle B\langle K\rangle B=B\langle J\rangle\langle K\rangle B$. In particular, $P_{J}=B\langle J\rangle B$ is a subgroup of $G$, and $P_{R}=G, P_{\emptyset}=B$;

(iii) if $w_{1}, w_{2} \in W$ satisfy $w_{1} \neq w_{2}$, then $B w_{1} B \neq B w_{2} B$;

(iv) if $J, K, L \subseteq R$, then $P_{J} \cap\left(P_{K} P_{L}\right)=\left(P_{J} \cap P_{K}\right)\left(P_{J} \cap P_{L}\right)=P_{J \cap K} P_{J \cap L}$.

Proof We first show that $R$ consists of involutions in $W$. Let $r \in R$. Applying (iii) with $w=r^{-1}$ yields $B r B r^{-1} B \subseteq B r^{-1} B \cup B$. In view of (iv) and $B \subseteq B r B r^{-1} B$, this implies

$$
B r B r^{-1} B=B r^{-1} B \cup B \text {. }
$$

Inverting the sets at both sides of the equation, we get $B r B r^{-1} B=B r B \cup B$, which, again by use of (iv), together with (1) leads to

$$
B r B=B r^{-1} B
$$

Applying (iii) with $w=r$ shows $B r B r B \subseteq B r B \cup B r^{2} B$. On the other hand, (1) and (2) give

$$
B r B r B=B r B r^{-1} B=B r B \cup B \text {. }
$$

Thus, we must have $B=B r^{2} B$, i.e. $r^{2} \subseteq B$. Since $r^{2} \subseteq N$, by definition, we derive $r^{2}=H$, so $r^{2}=1 \in W$. Since $r=1$ would contradict (iv), it follows that $r$ is an involution of $W$. An immediate consequence (inversion of (iii)) is

$$
w B r \subseteq B w B \cup B w r B \text { for all } r \in R \text { and } w \in W .
$$


We now prove

(ii) Obviously, $B\langle J\rangle B\langle K\rangle B \supseteq B\langle J\rangle\langle K\rangle B$. We next show $B\langle J\rangle B\langle J\rangle\langle K\rangle B \subseteq B\langle J\rangle B\langle K\rangle B$. Let $g \in B\langle J\rangle B\langle J\rangle\langle K\rangle B$. Then there are $r_{1}, \ldots, r_{q} \in J$ such that $g \in B r_{1} \cdots r_{q} B\langle J\rangle\langle K\rangle B$. If $q=0$, then $g \in B$ and there is nothing to prove. Otherwise, we have

$$
\begin{aligned}
B r_{1} \cdots r_{q} B\langle J\rangle\langle K\rangle B & \subseteq B r_{1} \cdots r_{q-1} B r_{q}\langle J\rangle\langle K\rangle B \\
& \subseteq B r_{1} \cdots r_{q-1} B\langle J\rangle\langle K\rangle B
\end{aligned}
$$

by axiom (iii), whence $g \in B r_{1} \cdots r_{q-1} B\langle J\rangle\langle K\rangle B$. By induction on $q$, it follows that $B\langle J\rangle B\langle J\rangle\langle K\rangle B \subseteq B\langle J\rangle\langle K\rangle B$. But then $B\langle J\rangle B\langle K\rangle B \subseteq B\langle J\rangle B\langle J\rangle\langle K\rangle B \subseteq B\langle J\rangle\langle K\rangle B$, and the first statement of (i) is proved.

Now $P_{J}$ is clearly nonempty and closed under taking inverses. From what we have just seen, $P_{J}$ is also closed under multiplication, so it is a subgroup. Finally, $P_{\emptyset}=B 1 B=B$, and $P_{R}=B R B=B N B=\langle B, N\rangle=G$, whence (ii).

(iii) Suppose $w_{1}, w_{2} \in W$ with $w_{1} \neq w_{2}$. Without loss of generality, we may assume $l\left(w_{1}\right) \leq l\left(w_{2}\right)$. If $l\left(w_{2}\right)=0$, then $B w_{1} B=B w_{2} B$ would imply $w_{1} \subseteq B \cap N=H$, whence $w_{1}=1=w_{2}$, a contradiction. Thus $B w_{1} B \neq B w_{2} B$ and we are done. Let $l\left(w_{2}\right) \leq 1$. Then there is an involution $r \in R$ such that $l\left(r w_{2}\right)<l\left(w_{2}\right)$. By induction on $l\left(w_{2}\right)$ we have $B r w_{2} B \neq B w_{1} B, B r w_{1} B$, so $B r w_{2} B \cap B r B w_{1} B=\emptyset$. Now $B w_{1} B=B w_{2} B$ would imply $B r w_{2} B \cap B r B w_{2} B=\emptyset$, which is absurd as $B r w_{2} B$ is contained in this intersection. Hence $B w_{1} B=B w_{2} B$, establishing (iii).

(i) For $r \in R$, set $C_{r}=\{w \in W \mid B r B w B=B r w B\}$. We first prove two claims on these $C_{r}$.

$$
C_{r} \cap r C_{r}=\emptyset
$$

Suppose $w \in C_{r}$. Then $B r B r w B=B r B r B w B=B w B \cup B r w B$, so $r w \notin C_{r}$, and $w \notin r C_{r}$, settling (5).

If $w \in C_{r}$ and $s \in R$ with $w s \notin C_{r}$, then $r w=w s$.

For,

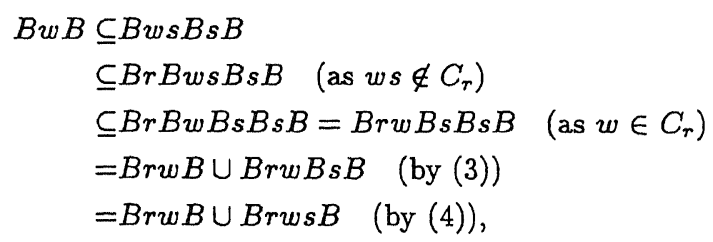

so $w \in\{r w, r w s\}$ by (iii). But $w=r w$ conflicts with $r \neq 1$, so $w=r w s$. This yields $r w=w s$ as required for (6).

Since, clearly $1 \in C_{r}$, we have obtained the Hyperplane Condition (vi) of 4.1, so Theorem 4.1 ends the proof of (i). 
(iv) Observe

$$
\begin{aligned}
P_{J} \cap\left(P_{K} P_{L}\right) & =B\langle J\rangle B \cap B\langle K\rangle B\langle L\rangle B= \\
& =B\langle J\rangle B \cap B\langle K\rangle\langle L\rangle B \quad \text { (by (ii)) } \\
& =B(\langle J\rangle \cap(\langle K\rangle\langle L\rangle)) B \quad \text { (by (iii)) } \\
& =B(\langle J\rangle \cap\langle K\rangle)(\langle J\rangle \cap\langle L\rangle) B \quad \text { (by (i) and 5.2.) } \\
& =B(\langle J\rangle \cap\langle K\rangle) B B(\langle J\rangle \cap\langle L\rangle) B \quad \text { (by (ii)) } \\
& =(B\langle J\rangle B \cap B\langle K\rangle B)(B\langle J\rangle B \cap B\langle L\rangle B) \quad \text { (by (iii)) } \\
& =\left(P_{J} \cap P_{K}\right)\left(P_{J} \cap P_{L}\right) .
\end{aligned}
$$

But also

$$
B(\langle J\rangle \cap\langle K\rangle) B B(\langle J\rangle \cap\langle L\rangle) B=B\langle J \cap K\rangle B B\langle J \cap L\rangle B=P_{J \cap K} P_{J \cap L}
$$

in view of 5.2 (ii) and (i).

This ends the proof of the theorem.

7.16 Definition Let $(B, N, W, R)$ be a Tits system in a group $G$. Then according to the theorem $(W, R)$ is a Coxeter system, so there exists a Coxeter matrix $M=\left(m_{r, s}\right)_{r, s \in R}$ such that $(W, R)$ is of type $M$. We shall also refer to $M$ as the type of the Tits system. The chamber system $\mathcal{C}(B, N, W, R)$ associated with the Tits system is the chamber system over $R$ whose chambers are the cosets $g B$ for $g \in G$ and in which, for each $e \in R$, the chambers $g B$ and $h B$ are $r$-adjacent if and only if $B h^{-1} g B \subseteq B\langle r\rangle B$. Observe that this is indeed a chamber system because if $g B, h B$ and $h B, k B$ are $r$-adjacent pairs, then by (ii) of the theorem $B k^{-1} g B \subseteq B k^{-1} h B h^{-1} g B \subseteq B\langle r\rangle B\langle r\rangle B=B\langle r\rangle B$.

7.17 Corollary Let $\mathcal{C}$ be the chamber system associated with the Tits system $(B, N, W, R)$ in a group $G$. Then

(i) $\mathcal{C}$ is a thick building of type $M$;

(ii) the group $G$ acts as a group of type automorphisms (by left multiplication) on $\mathcal{C}$, which is transitive on set of all incident chamber, apartment pairs.

Proof (i) The chamber system $\mathcal{C}$ is of type $M$. For, if $r, s$ are distinct elements of $R$, then the $\{r, s\}$-cell containing $c=B$ consists of all chambers in $B W_{\{r, s\}} B$, and by use of Theorem 7.16 , it is readily seen that the chamber system induces a generalized $m_{r, s}-$ gon on this cell.

Next, if $B, b r_{1} B, b_{1} r_{1} b_{2} r_{2} B, \ldots, b_{1} r_{1} b_{2} r_{2} \cdots b_{t} r_{t} B=B$ is a simple closed path with minimal type $r_{1} \cdots r_{t}$, then, by 11.6.4 (i), $B=B r_{1} b_{2} r_{2} \cdots b_{t} r_{t} B=B r_{1} r_{2} \cdots r_{t} B$, so that by 11.6 .4 (iii), $r_{1} r_{2} \cdots r_{t}=1$, proving that the only simple closed path starting at $c$ with minimal type is the trivial path. Since $G$ is transitive on $\mathcal{C}$, this establishes (i).

(ii) Clearly, $G$ is transitive on the set of chambers, and the stabilizer of the chamber $B$ coincides with $B$. Set $A=N B / B$. Then $A$ inherits the chamber system structure from $\mathcal{C}$ by restriction. The map $W=N / H \rightarrow A$ leads to an isomorphism of chamber systems, and so establishes that $A$ is isomorphic to $\mathcal{C}(W, R)$. Furthermore, $N$ stabilizes $A$ and $H$ fixes $A$ pointwise, so there is an action of $W=N / H$ on $A$. The above isomorphism then gives that $N$ acts transitively on $A$. 
7.18 Examples (i) Let $G$ be a doubly transitive permutation group on a set $\Omega$. Pick two (distinct) points $c, c^{\prime} \in \Omega$ and set $A=\left\{c, c^{\prime}\right\}$. Set $B=G_{c}$ and $N=G_{A}$. Then $B \cap N=G_{c, c^{\prime}}$ has index 2 in $N$. Thus $N /(B \cap N) \cong \mathbb{Z} /(2)$. Using $G=B \cup B r B$ where $r \in N$ induces the transposition $\left(\omega, \omega^{\prime}\right)$ on $A$ (the existence of $r$ is equivalent to double transitivity of $G$ on $\Omega$ ) it is readily seen that $B, N$ gives rise to a Tits system of type $A_{1}$.

(ii) Let $G=G L_{n}(\mathbb{F})$ for some field $\mathbb{F}$. Taking $B$ to be the subgroup of $G$ consisting of all invertible upper-diagonal matrices and $N$ the subgroup of all invertible monomial matrices, we obtain a Tits system of type $A_{n-1}$. The geometry of the corresponding chamber system is the projective geometry of rank $n-1$.

(iii) For a nondegenerate polar space of finite rank $n$ all of whose lines have at least three points, it is possible to prove in a rather elementary way that the automorphism group satisfies the conditions of Proposition 7.13 in its action on the chamber system described in Example 7.5(vi), thus giving rise to a Tits system of type $B_{n}$. This is however an instance of the following highlights.

7.19 Theorem Let $\mathcal{C}$ be a thick building of type a connected diagram $M$ of rank $n<\infty$.

(i) (Tits' classification of buildings of spherical type) If $n \geq 3$ and $W(M)$ is finite, then $\mathcal{C}$ corresponds to a Tits system and is known.

(ii) (Bruhat-Tits' classification of buildings of affine type) If $n \geq 4$ and $W(M)$ is affine, then $\mathcal{C}$ corresponds to a Tits system and is known.

Here 'known' means that, for given $\mathcal{C}$ as in the theorem, there is an explicitly known construction of a building $\mathcal{C}^{\prime}$ in terms of algebraic objects (e.g., a field possibly an extension field, and a vector space, and in (ii) a valuation on a field) such that $\mathcal{C} \cong \mathcal{C}^{\prime}$.

The proof of (i) is in [Tits 1974]; for the proof of (ii), see [BrTi 1984]. There are, however far more buildings than those classified by the above theorem. In [Ron 1989] a description of very general constructions is given.

See [BCN 1989] for an overview of synthetic properties of the geometries $G / P$ for $P$ a maximal subgroup of the group $G$ containing the subgroup $B$ of a Tits system in $G$. The Bruhat order is closely related to the (Zariski) topological structure of reductive algebraic groups: The closure of $B w B$ in $G$ is the union of all $B x B$ for $x \in \operatorname{Bruh}(w)$. The notion of shelling, related to the Bruhat order, has been used to analyze the topological nature of buildings, see [BjWa 1982]. In [LakSe 1986] the structure (regarding homogeneous generators etc.) of the ring of polynomial functions on $G / P$ is studied.

\section{Some algorithmic issues}

Both from the pure geometric and the Lie group theoretic it is desirable to be able to compute and to compute efficiently with Coxeter group elements. We shall devote some attention to the word problem and to computations in the Reflection Representation.

8.1 The word problem In view of Theorem 5.3, the word problem for Coxeter groups is solvable. For, the following set of rewrite rules provides a solution to the problem of deciding, when given a word $\mathbf{i} \in I^{*}$ whether $\rho(\mathbf{i})=1$.

$$
\begin{aligned}
i i & \Rightarrow 1 \quad(1 \leq i \leq n), \\
i j i \cdots & \Rightarrow j i j \cdots \quad(1 \leq i, j \leq n) \quad \text { [both sides of length } m_{i j} \text { ] }
\end{aligned}
$$


According to Tits' algorithm, one applies the rewrite rules of the second kind to i till one of letters $i \in I$ appears in $\mathbf{i}$ twice in a row. If it never happens (verifiable in finitely many steps) the resulting word is reduced and the answer is yes if the result is the empty word and no otherwise. If it does happen, remove $i i$ from the word - a rewrite rule of the first kind - and repeat the procedure (the length of the word has decreased, so termination is guaranteed).

Let us focus on the complexity of this algorithm. As before, for $w \in W$, let $I_{w}^{*}$ be the set of all minimal words in $I^{*}$ representing $w$. The number $\operatorname{red}(w)=\left|I_{w}^{*}\right|$ gives a lower bound for the complexity of Tits' algorithm. By [Stan 1984], for $W=W\left(A_{n-1}\right) \cong \operatorname{Sym}_{n}$, the number $r e d\left(w_{0}\right)$ equals the number of standard Young Tableaux of staircase shape (that is, one row of each length $<n$ ) for which, in turn, a closed expression has been found:

$$
\operatorname{red}\left(w_{0}\right)=\frac{\left(\begin{array}{l}
n \\
2
\end{array}\right) !}{1^{n-1} 3^{n-2} 5^{n-3} \cdots(2 n-1)^{0}} .
$$

Using Stirling: $n ! \approx \sqrt{2 \pi n}\left(\frac{n}{e}\right)^{n}$, we see that the expression in the $e$ exponent grows as

$$
\left(\frac{1}{2}+\left(\begin{array}{l}
n \\
2
\end{array}\right)\right) \log \left(\begin{array}{l}
n \\
2
\end{array}\right)-\left(\begin{array}{l}
n \\
2
\end{array}\right)-\sum_{k=1}^{n}(n-k) \log (2 k-1) \geq\left(\begin{array}{l}
n \\
2
\end{array}\right) \log \frac{n}{12}
$$

so that the complexity is at least $(n / 12)^{\left(\begin{array}{c}n \\ 2\end{array}\right)}$ in terms of $n=\min \left(|R|, l\left(w_{0}\right)\right)$. Faster methods are available through the Reflection Representation.

The action of a matrix on a vector involves $n^{3}$ operations (with more sophistication, the exponent can be brought down to a number exceeding 2). But the action of a reflection involves only an inner product computation and a vector addition, so is linear in $n$. Hence just $n l$ operations are needed for a word $\mathrm{i} \in I^{*}$ of length $l$ to compute the image $\rho(\mathrm{i}) v$ of a vector $v \in \mathbb{R}^{n}$.

8.2 Algorithm Computing a minimal word representing $w$

Reduce

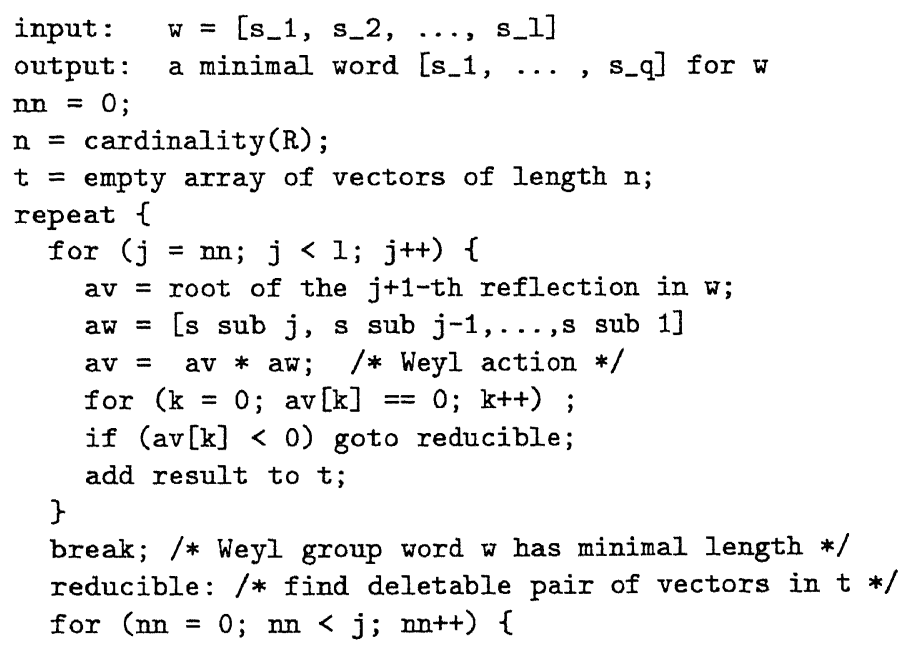




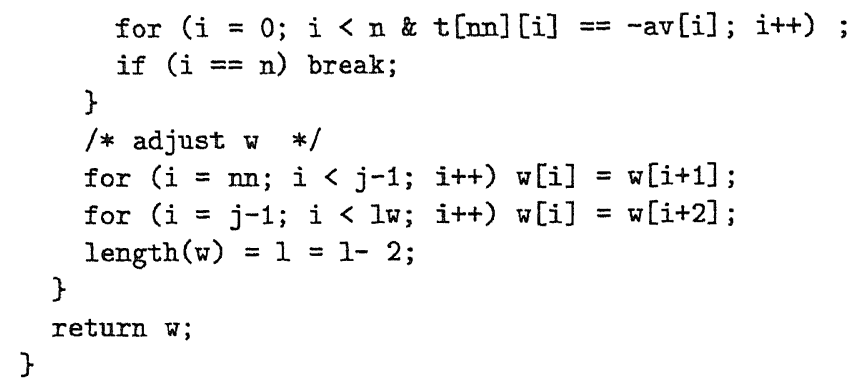

A blend of 4.1(ii) and 5.1(i) can be used to prove correctness of this algorithm.

8.3 Rewrite systems In order to compare elements of $I^{*}$, we introduce a linear ordering $<$ on $I^{*}$ refining the relation | ('is a divisor of'). For all $\mathbf{i}, \mathbf{i}^{\prime}, \mathbf{i}^{\prime \prime}, \mathbf{j} \in I^{*}$ we require

(i) if $\mathbf{i} \neq \epsilon$ then $\epsilon<\mathbf{i}$;

(ii) if $\mathbf{i}^{\prime}<\mathbf{i}^{\prime \prime}$ then $\mathbf{i} \mathbf{i}^{\prime} \mathbf{j}<\mathbf{i} \mathbf{i}^{\prime \prime} \mathbf{j}$.

A linear ordering with these properties is called a reduction ordering. The total degree lexicographic ordering (first according to total degree, then lexicographically) is an example. The ordering is Noetherian in the sense that each strictly decreasing sequence terminates (after finitely many steps). A rewrite rule has the form uiv $\Rightarrow$ ui'v with $\rho(\mathbf{i})=\rho\left(\mathbf{i}^{\prime}\right) \in W$ and $\mathbf{i}>\mathbf{i}^{\prime}$. We say that $\mathbf{i}$ reduces to $\mathbf{i}^{\prime}$ via $\mathcal{R}$ if there is a sequence of rewrite rules from $\mathcal{R}$ that, when successively applied to $i$, yield $i^{\prime}$. In order to solve the word problem for the Coxeter system $(W, R)$, it suffices to find a finite set $\mathcal{R}$ of rewrite rules such that any $\mathbf{i} \in I^{*}$ reduces to the unique minimal element of $\rho^{-1}(\rho(i))$ via $\mathcal{R}$. Such a system is called confluent.

8.4 Examples (i) For $M=H_{3}$, the following system $\mathcal{R}$ of 7 rewrite rules is confluent with regard to the total degree-reduction ordering satisfying $1<2<3$.

$$
\begin{gathered}
11 \Rightarrow \epsilon ; 22 \Rightarrow \epsilon ; 33 \Rightarrow \epsilon \\
212 \Rightarrow 121 ; 31 \Rightarrow 13 ; 3232 \Rightarrow 2323 ; \\
321321 \Rightarrow 232132 .
\end{gathered}
$$

(ii) $M=A_{n}$, so $W=\operatorname{Sym}_{n+1}$. A confluent system is given by

$$
\begin{cases}i i \Rightarrow \epsilon & i=1, \ldots, n+1 \\ i j \Rightarrow j i & 1 \leq j \leq i-2 \leq n-1 \\ i i-1 i \cdots j i \Rightarrow i-1 i i-1 \cdots j+1 j & 1 \leq j<i \leq n+1\end{cases}
$$

$n^{2}-n$ equations in total.

(iii) There exists an infinite confluent set of rewrite rules with respect to any reduction ordering: for each instance $r_{2} \cdots r_{q} r=r_{1} \cdots r_{q}$ of the exchange condition a rewrite rule can be obtained by turning the side of the equation containing the least expression into the right hand side of the rewrite rule; take $\mathcal{R}$ to be the collection of all rewrite rules obtained in this way together with all rewrite rules of the form $i i \Rightarrow \epsilon$. 
8.5 Problem Find an efficient complete (possibly infinite) set of rewrite rules for any Coxeter group. See [LeCh 1986] for an answer in the special case where $m_{i, j}>2$ for each $i \neq j$. The solution is:

$$
\begin{cases}i i \Rightarrow \epsilon & i=1, \ldots, n+1 \\ a_{1} \cdots a_{l} l a s t\left(a_{l}^{-1}\right) \Rightarrow \sec \left(a_{1}\right) a_{1} \cdots a_{l} & a_{r}=\mathrm{k}_{i_{r} j_{r}}(1 \leq r \leq l)\end{cases}
$$

where $\operatorname{first}\left(a_{1}\right)>\sec \left(a_{1}\right)$, and, for all $r \in\{2, \ldots, n-1\}, \sec \left(a_{r}\right)>\operatorname{first}\left(a_{r}\right), \operatorname{last}\left(a_{r}\right) \neq$ first $\left(a_{r+1}\right), \sec \left(a_{r+1}\right)=\operatorname{last}\left(a_{r}^{-1}\right)$.

8.6 Problem The conjugacy problem is the quest for an algorithm to determine, for each input $\mathbf{i}, \mathbf{i}^{\prime} \in I^{*}$, whether $\rho(\mathbf{i})$ and $\rho\left(\mathbf{i}^{\prime}\right)$ are conjugate. The problem has a solution for Coxeter systems of type $M=\left(m_{i, j}\right)$ with $m_{i, j} \geq 4$ for all $i, j \in I$ with $i \neq j$, see [ApSc 1983], [Appel 1984], and [Bezv 1986].

8.7 Generating the elements of $W$ In various applications, it is useful to be able to generate all elements of the Coxeter group, or those of length bounded by a certain number $N$. Suppose we have generated a list of words $I_{N-1}^{*}$ representing all elements of length $N-1$. Also assume that for each $\mathrm{i} \in I_{N-1}^{*}$, we have recorded all pairs $(j, \mathrm{x}) \in I \times I_{N-2}^{*}$ for which $\rho(\mathbf{i} j)=\rho(\mathbf{x})$. Then each $j \in I$ for which there is no recorded pair of shape $(j, \mathbf{x})$ will provide a representative $\mathrm{i} j$ of an element of length $N$, together with the record $(j, \mathrm{i})$. It may happen however that two newly generated words, say $\mathbf{i} j$ and $\mathbf{i}^{\prime} j^{\prime}$ satisfy $\rho(\mathbf{i} j)=\rho\left(\mathbf{i}^{\prime} j^{\prime}\right)$. But then 5.3.(i) gives the existence of $\mathrm{y} \in I_{N-m}^{*}$, where $m=m_{j, j^{\prime}}$ such that $\mathrm{i} j=\mathrm{yk}_{j, j^{\prime}}^{-1}$ and $\mathbf{i}^{\prime} j^{\prime}=\mathrm{yk}_{j^{\prime}, j}^{-1}$ (cf. $\S 5$ for the definition of $\mathbf{k}_{i, j}$ ), so that the recorded data helps to detect the double occurrences of representatives for $\rho(i j)$.

Another conceivable way to run through the elements can be furnished by a Hamiltonian path in the graph $(\mathcal{C}(W, R), \sim)$, that is a path meeting every vertex once. In [CSW 1989], Hamilton circuits (paths ending in a neighbor of the starting vertex) for all finite Coxeter groups are exhibited.

8.8 Generating the weights in a $W$-orbit As we shall see in the next section, some computations regarding Lie group representations require the generation of a whole Weyl group orbit of vectors in the Reflection Representation $V$ of $W$. As the Weyl group can become very large, it would not be wise to store an entire orbit (a regular orbit of $W\left(E_{8}\right)$ has 696729600 elements!). In many cases however, it is not necessary to store the entire orbit; all that is needed is to enumerate the vectors one by one.

The case $M=A_{n}$ is particularly easy. In fact, the root system can be embedded in $\mathbb{R}^{n+1}$ in such a way that $W\left(A_{n}\right) \cong \operatorname{Sym}_{n+1}$ acts by permutations of the coordinates. It follows that after a suitable linear transformation, the calculation of the $W$-orbit can be done simply by generating all permutations of a given finite sequence of integers. Using lexicographical order, it is not difficult to run through all elements of the orbit using very little memory. The other classical groups $\left(B_{n}\right.$ and $\left.D_{n}\right)$ can be dealt with in a similar manner; the Weyl group operates by permutations of coordinates and certain sign changes. Again we can use a lexicographical order to enumerate all vectors of the orbit.

For the types $M=G_{2}, F_{4}, E_{6}, E_{7}, E_{8}$, the above principle can be used on a suitable subgroup. By way of example, we take $M=E_{7}$. Consider the image $w \in W\left(E_{7}\right)$ under $\rho$ of the word 134562453413245676543245613452431 . The subgroup of $W\left(E_{7}\right)$ generated by $w$ and $\rho_{i}$ for $i \in\{1,3,4,5,6,7\}$ is a reflection subgroup, whence a Coxeter group (cf. 5.4). Its type is $A_{7}$. The index of this Weyl subgroup $W\left(A_{7}\right)$ is 72 . Taking $t=\rho_{1} \rho_{2} \rho_{3} \rho_{4} \rho_{5} \rho_{6} \rho_{7}$, a socalled Coxeter element (cf. [Bourb 1968]) of $E_{7}$, and $c=\rho_{2} \rho_{4} \rho_{3} \rho_{5} t$, it can be shown that the 
set $X=\left\{1, c, c^{2}, c^{5}\right\}$. $\langle t\rangle$ is a full set of coset representatives. Thus, for any vector $v \in V$, we have $W v=W\left(A_{7}\right) X v$. Consequently, starting with $X v$, we first prevent double occurrences by creating the set $Y$ of all elements that are minimal (with respect to the lexicographical ordering in $\mathrm{IR}^{8}$ used above) in $W\left(A_{7}\right) x v$ for some $x \in X$. Next we enumerate the elements of $W\left(A_{7}\right) y$ for each $y \in Y$. This principle has been implemented in the software package $\mathrm{LiE}$, built at CWI, Amsterdam.

\section{Representation Theory of Lie groups}

The Weyl groups are the backbone in the structure theory of semi-simple Lie groups. In this section we briefly review how they are used in the finite-dimensional rational linear representation theory. The decompositions of restrictions of representations to a closed reductive Lie subgroups will be our main topic.

9.1 The basic structure of reductive Lie groups The reductive Lie groups and Lie algebras are well described in various text books, e.g. [Hum 1974], [Var 1984], [Ser 1987]. There are tight relations with the representation theory for reductive algebraic groups in characteristic 0, see [Spr 1981] and [Jantz 1987]. For algebraic groups of arbitrary characteristic the representation is far more complicated. Here we shall only deal with groups defined over the field of complex numbers.

Let $G$ be a connected reductive complex Lie group. Fix a maximal torus $T$ and a Borel subgroup $B$ of $G$ containing $T$. (All tori are conjugate in $G$, and so are all Borel subgroups). Then its derived group $[G, G]$ is semi-simple and its center $Z=Z(G)$ is contained in $T$, while $G=Z \cdot[G, G]$, and $T=(T \cap[G, G]) . Z$. We shall restrict our attention to the case where $[G, G]$ is simply connected, $Z^{(0)}$ is the connected component of 1 in $Z$ and $G=Z^{(0)} \times[G, G]$. This may always be achieved by replacing $G$ by a suitable cover. The group $B$ gives rise to a Tits system $(B, N, W, R)$ in $G$, where $N=N_{G}(T)$.

Let $V$ be a finite-dimensional complex rational representation of $G$. Since $T$ is a torus, there is a basis of $V$ with respect to which every element of $T$ has diagonal form. Thus the restriction to $T$ of the character of $G$ on $V$ is a sum of $\operatorname{dim} V$ rational linear (i.e., 1dimensional) characters. The rational linear characters of $T$ form a commutative group $\Lambda(T)$ (written additively); they are usually called weights of $G$ with respect to $T$. Note that $\Lambda(T)$ is actually a free $\mathbb{Z}$-module of $\operatorname{rank} r$, where $r=\operatorname{dim} T$, the Lie rank of $G$. We shall denote by $t^{\lambda}$ the image in $\mathbb{C}$ of $t \in T$ under $\lambda \in \Lambda(G, T)$. The normalizer $N$ of $T$ in $G$ permutes the characters of $T$ pertaining to any representation of $G$, and the kernel of the action of $N$ on $\Lambda(T)$ coincides with $T$, so $W=N / T$ acts faithfully on $\Lambda(G, T)$. After tensoring with IR, the additive group $\Lambda(T)$ becomes a real vector space on which $W$ acts linearly. There is a natural decomposition $\Lambda(T)=\Lambda\left(Z^{(0)}\right) \oplus \Lambda\left(T_{s}\right)$, where $T_{s}=T \cap[G, G]$; the group $W$ acts trivially on $\Lambda\left(Z^{(0)}\right)$ and via the Reflection Representation on $T \cap[G, G]$. Now consider the Lie algebra $\mathbf{g}$ of $G$. The group $G$ acts on $\mathbf{g}$ via the so-called adjoint representation. The set of all nonzero weights $\lambda \in \Lambda$ whose eigenspaces $\mathbf{g}_{\lambda}=\left\{v \in \mathbf{g} \mid v t=v\left(t^{\lambda}\right)\right.$ for all $\left.t \in T\right\}$ are nontrivial is the root system of $G$ with respect to $T$, notation $\Phi(G, T)$, or just $\Phi$. It coincides with the root system $\Phi$ that arose from the Reflection Representation in Theorem 4.1. The eigenspace $\mathbf{t}=\mathbf{g}_{0}$ with the trivial weight 0 is the Lie subalgebra corresponding to $T$.

The subgroup $B$ of $G$ corresponds to the Borel subalgebra $\mathbf{b}$ of $\mathbf{g}$ spanned by $\mathbf{t}$ and all $\mathbf{g}_{\alpha}$ for $\alpha>0$ in the ordering of 4.1 (ii). The roots $\alpha \in \Phi$ with $\alpha>0$ corresponding to the reflections $r \in R$ are called the fundamental roots (with respect to $B$ ). Given two 
weights $\lambda, \mu$, we write $\lambda \leq \mu$ to indicate that $\mu-\lambda$ is a non-negative linear combination of fundamental roots. This is consistent with the choice of the ordering from 4.1(ii). Suppose for the sake of presentation that $G$ is semisimple, so that $T=T_{s}$. The weight lattice $\Lambda(G, T)$ has a basis $\omega_{1}, \ldots, \omega_{n}$ such that $2 B\left(\omega_{i}, \alpha_{j}\right) / B\left(\alpha_{j}, \alpha_{j}\right)=\delta_{i, j}$. This is the so-called basis of fundamental weights. The span $\mathbb{I N} \omega_{1}+\ldots+\mathbb{I N} \omega_{n}$ is denoted by $\Lambda^{+}(G, T)$. Its members are called dominant weights. If $V$ is irreducible, it has a unique highest weight (with respect to $<$ ), with multiplicity 1 . Conversely, for each $\lambda \in \Lambda^{+}(G, T)$, there is a unique rational finite-dimensional complex representation $V$ with highest weight $\lambda$ (up to isomorphism, of course); it is denoted by $V(\lambda)$.

Let $H$ be a reductive closed Lie subgroup of $G$. Denote by $n$ and $m$ the Lie ranks of $G$ and $H$, respectively. The fact that $H$ is reductive ensures that any finite-dimensional rational representation of $H$ decomposes into a direct sum of irreducibles. Branching or restriction is the decomposition into irreducibles of a representation of $H$ that is obtained by restriction from a highest weight module of $G$. Let $S$ be a maximal torus of $H$. Then there is a maximal torus $T$ of $G$ containing $S$. Thus, for a weight $\lambda=\left(\lambda_{1}, \ldots, \lambda_{n}\right)$ of $G$, and a weight $\mu=\left(\mu_{1}, \ldots, \mu_{m}\right)$ of $H$, branching is the determination of the multiplicity $\left(V(\mu),\left.V(\lambda)\right|_{H}\right)$ of the representation $V(\mu)=V(H, T, \mu)$ in $\left.V(G, S, \lambda)\right|_{H}$. Let $X_{1}, \ldots, X_{n}$ be indeterminates, and write $X^{\lambda}$ for the monomial $X_{1}^{\lambda_{1}} \cdots X_{n}^{\lambda_{n}}$; thus $X^{\omega_{i}}=X_{i}$. We want to find an explicit description of

$$
P_{G \downarrow H}(X, \lambda):=\sum_{\mu}\left(V(\mu),\left.V(\lambda)\right|_{H}\right) X^{\mu} .
$$

In the case of the very special reductive subgroup $H=T$, an explicit form is known. Write

$$
\delta(\Phi)=\frac{1}{2} \sum_{\alpha \in \Phi^{+}} \alpha
$$

so that $\delta(\Phi)=\sum_{i=1}^{n} \omega_{i}$. It is convenient to parametrize the elements of $T$ by the variables $X_{1}, \ldots, X_{n}$ in such a way that $\left(h\left(X_{1}, \ldots, X_{n}\right)\right)^{\lambda}=X^{\lambda}$ for $\lambda \in \Lambda^{+}$.

9.2 Theorem (Weyl's Character Formula) For each $\lambda \in \Lambda^{+}(G, T)$, the character of $T$ (as a function of $X$ ) on the $G$-module $V(\lambda)$ is

$$
P_{G \downarrow T}(X, \lambda)=\frac{\sum_{w \in W^{\prime}} \operatorname{sgn}(w) X^{w(\delta+\lambda)}}{X^{\delta} \prod_{\alpha \in \Phi^{-}}\left(1-X^{\alpha}\right)}
$$

where $\delta=\delta(\Phi)$

Note that $\Lambda^{+}(T, T)=\Lambda(G, T)$. Thus, in $P_{G \downarrow}(X, \lambda)$ we should expect negative powers of $X$.

9.3 Example $G=A_{1}$. Then $\Lambda^{+}(G, T) \cong \mathbb{N}$ and

$$
P_{G \downarrow T}(X, m)=X^{m}+X^{m-2}+\ldots+X^{2-m}+X^{-m} \quad(m \in \mathbb{I N}) .
$$

Let res : $\Lambda(G, T) \rightarrow \Lambda(H, S)$ denote the restriction map. The Borel subgroup of $H$ can be chosen in such a way that, res $(\alpha) \notin \Phi(H, T)^{-}$for each $\alpha \in \Phi(G, T)^{+}$. Let 
$\Phi_{0}=\{\alpha \in \Phi \mid \operatorname{res}(\alpha)=0\}, \Phi(G, T)^{+}=\Phi^{+}=\Phi \cap \Lambda^{+}(G, T)$ and $W_{0}$ the subgroup of $W$ generated by the reflections with roots in $\Phi_{0}$. Then $W_{0}$ is readily seen to be generated by fundamental reflections. Thus, by 5.1 (iv), each coset in $W / W_{0}$ has a unique representative in $W$ of minimal length, the set of these representatives is denoted by $W^{0}$. Write $A$ for the multiset $\operatorname{res}\left(\Phi(G, T)^{+} \backslash \Phi_{0}\right) \backslash \Phi(H, S)^{+}$supplied with the multiplicities $m_{\alpha}=$ $\left|\left(\Phi(G, T)^{+} \backslash \Phi_{0}\right) \cap \operatorname{res}^{-1}(\alpha)\right|-\left|\Phi(H, S)^{+} \cap\{\alpha\}\right|$ for $\alpha \in A$, and let $L$ be the lattice of integral non-negative linear combinations of elements in $A$. Kostant's partition function $p_{A}$ on $L$ is given by

$$
\frac{1}{\prod_{\alpha \in A}\left(1-X^{\alpha}\right)^{m_{\alpha}}}=\sum_{\beta \in L} p_{A}(\beta) X^{\beta}
$$

its domain of definition is extended to $L \otimes \mathbb{R}$ by putting $p_{A}(\beta)=0$ if $\beta \notin L$. Finally put $\delta_{0}=\delta\left(\Phi_{0}\right)$ and

$$
D(\lambda)=\prod_{\alpha \in \Phi_{0}^{+}} \frac{(\lambda, \alpha)}{\left(\delta_{0}, \alpha\right)}
$$

9.4 Theorem (cf. [Heckman 1980])

$$
\left(V(\mu),\left.V(\lambda)\right|_{H}\right)=\sum_{w \in W^{0}} \operatorname{sgn}(w) D(w(\lambda+\delta)) p_{A}(\operatorname{res}(w(\lambda+\delta))-(\mu+\operatorname{res}(\delta))) .
$$

The theorem can be proved using Weyl's Character Formula; conversely Weyl's Formula is a special case of the theorem. In using this formula for obtaining an explicit answer, one needs to enumerate the elements of Weyl group orbits.

The book [ $\mathrm{McPa} 1981]$ is almost entirely devoted to explicit information of this kind.

9.5 Example (i) Let $G$ be a Lie group of type $G_{2}$, with root system $\Phi$ and fundamental roots $\beta_{1}, \beta_{2}$, where $\beta_{1}$ is long and $\beta_{2}$ is short. There is a subgroup $H$ of type $A_{2}$, whose root system $\Phi(H, T)$ consists of the long roots of $\Phi(G, T)$; its fundamental roots are $\alpha_{1}=\beta_{1}$ and $\alpha_{2}=\beta_{1}+3 \beta_{2}$. We want to give the formal power series $P_{G \downarrow H}(x, y)=$ $\sum_{\lambda, \mu}\left(V(\mu),\left.V(\lambda)\right|_{H}\right) x^{\lambda_{1}} y^{\lambda_{2}} z^{\mu_{1}} u^{\mu_{2}}$, where the sums is taken over all $\lambda \in \Lambda^{+}(G, T)$ and $\mu \in \Lambda^{+}(H, T)$. The restriction map with respect to the bases of fundamental weights is given by $\operatorname{res}(1,0)=(1,1)$ and $\operatorname{res}(0,1)=(0,1)$. Thus $\Phi_{0}$ is empty, $W_{0}=\{1\}, A$ is the set of positive short roots of $G$ and all multiplicities $m_{\alpha}$ are equal to 1 , so $D \equiv 1$ and $W^{0}=W$. The branching series of $G_{2}$ to the subgroup $A_{2}$ is (cf. [CoRu 1990]):

$$
P_{G \downarrow H}(x, y, z, u)=\frac{1-y x z u}{(1-y u)(1-x u)(1-y z)(1-y)(1-x z)(1-z u x)} .
$$

The formula in Theorem 9.2 is not the only closed form for characters. The Demazure Character Formula is another, cf. [Jantz 1987]. It is often more practical to list the multiplicities (i.e. coefficients of $X^{\lambda}$ ) of dominant weights $\lambda$ : as the character is $W$-invariant, the other multiplicities can be obtained from these by the action of $W$.

9.6 Theorem (Freudenthal's Weight Multiplicity Formula) Suppose $\lambda \in \Lambda^{+}(G, T)$. The multiplicity $m(\mu, \lambda)$ of the weight $\mu \in \Lambda(G, T)^{+}$in $V(\lambda)$ is determined by:

$$
m(\mu, \lambda)= \begin{cases}1 & \text { if } \lambda=\mu \\ 2 \frac{\sum_{\alpha \in \Phi}+\sum_{i=1}^{\infty} m(\mu+i \alpha, \lambda)(\mu+i \alpha, \alpha)}{(\lambda+\delta, \lambda+\delta)-(\mu+\delta, \mu+\delta)} & \text { if } \lambda<\mu \\ 0 & \text { otherwise }\end{cases}
$$


The formula enables one to compute all dominant weight multiplicities by recursion with respect to $<$, starting with the highest weight. Determination of all weights follows by computation of Weyl group orbits.

There is a converse to the determination of weights of a given representation: given a multiset $A$ of dominant weights, determine, if possible the multiset $B$ of dominant weights such that $A$ is the multiset of all dominant weights of the module $\bigoplus_{\beta \in B} m_{\beta} V(\beta)$. Such a procedure is called decomposition of $A$. Here is the most straightforward method.

9.7 Algorithm decompose $A$ : obtain a multiset $B$ such that $A$ is the set of dominant multiplicities of the module $\bigoplus_{\beta \in B} m_{\beta} V(\beta)$

() start with $B=\emptyset$;

(i) find a highest weight $\alpha \in A$; then $V(\alpha)$ must occur in $V$ with multiplicity $m_{\alpha}$, so add $\alpha$ to $B$ with multiplicity $m_{\alpha}$

(ii) set $D=$ dominant weights of $V(\alpha)$;

(iii) put $A=A \backslash D$ (multiset subtraction) and, if $A \neq \emptyset$, continue with (i).

It may happen that the multiplicities of the multisets involved become negative, in which case the algorithm still terminates and gives a virtual $G$-module decomposition (that is, possibly $m_{\beta}<0$ for some $\beta \in B$ ). This observation is of use in computations of symmetrized tensor product decompositions by means of Frobenius' Formula, which involves the decomposition of the multisets $p . A$ (for $p \in \mathbb{I N}$ ) consisting of all $p$-multiples of the members of a multiset $A$ of dominant multiplicities of a $G$-module.

A very crude way of computing the coefficient $\left(V(\mu),\left.V(\lambda)\right|_{H}\right)$ of the branching series $P_{G \downarrow H}(X, \lambda)$ is by first applying a routine (e.g. the one of 9.6$)$ to compute the multiset of dominant weights of the $G$-module $V(\lambda)$, next computing their restrictions to $S$ (using res) and then decomposing this multiset as an $H$-module (e.g., by 9.7). There is a vast literature on the question of how to do a better job in various special cases. Part of the explanation why it is feasible is that $P_{G \downarrow H}(X, \lambda)$ is a rational function in $X$ (cf. [CoRu 1990]) so that the behavior is determined by decompositions of the restrictions of $V(\lambda)$ 's for bounded $\lambda \in \Lambda^{+}$.

For generalizations of the classical formulas to the affine Lie groups, see, among others, [Kac 1985], [Mat 1988] and [Neid 1986].

9.8 Tensor product decompositions Tensoring is a special kind of branching, namely from $G \times G$ to the diagonal subgroup $H$ isomorphic to $G$. The most efficient formula known for the general case is

9.9 Klimyk's Formula (cf. [Hum 1968])

$$
P_{\otimes}(X, \lambda, \mu)=\sum_{\alpha \in A} m_{\alpha} t(\alpha+\mu+\delta) \overline{X^{\overline{\alpha+\mu+\delta}}-\delta} .
$$

where $\bar{\beta}$ is the unique weight in $W \beta \cap \Lambda^{+}(G, T), A$ is the multiset of dominant weights of $V(\lambda)$ with multiplicities $m_{\alpha}$, and $t(\mu)=(-1)^{l(w)}$ if $w \mu=\bar{\mu}$ and $\bar{\mu} \in \Lambda^{++}$(that is, all its coefficients on the basis $\omega_{1}, \ldots, \omega_{n}$ are nonzero), and 0 otherwise.

For another approach, see [Vret 1988].

9.10 Examples (i) $G=A_{1}$ and $d=2$. The Clebsch-Gordan formula reads $V(m) \otimes V(n)=$ $V(m+n) \oplus V(m+n-2) \oplus \ldots \oplus V(m-n)$ for all $m, n \in$ IN with $m \geq n$. 
(ii) $M=A_{n}$ The invertible map $\lambda \mapsto\left(\lambda_{1}+\cdots+\lambda_{n}, \lambda_{2}+\cdots+\lambda_{n}, \ldots, \lambda_{n}\right)$ sends each dominant weight to a partition of $d=\lambda_{1}+2 \lambda_{2}+\cdots+n \lambda_{n}$. Conversely, to each partition $\pi$ of a number $d$ we can assign an irreducible representation, also denoted by $\pi$, of $\operatorname{Sym}_{d}$ (cf. [JaKe 1981]). We fix a natural representation of $G$ on $\mathbb{C}^{n}$. The group $\operatorname{Sym}_{d} \times G$ acts on the homogeneous part $T^{d}\left(\mathbb{C}^{n}\right)$ of degree $d$ of the tensor algebra of $\mathbb{C}^{n}$ : the first component (isomorphic to $\mathrm{Sym}_{d}$ ) via permutation of the $d$ factors, the second diagonally on each of the $d$ factors. There is a unique irreducible $G$-module $V$ such that the $\operatorname{Sym}_{d} \times G$-module $\pi \otimes V$ occurs in $T^{d}\left(\mathbb{C}^{n}\right)$. It is the module with highest weight $\lambda$. Thus irreducible modules of $G$ are indexed by partitions. The Littlewood-Richardson rule (cf. [JaKe 1981], [Macd 1979]) is an algorithm that returns a certain multiset of partitions when given two partitions $\pi$ and $\sigma$. Translating input and output to weights, it gives the tensor product decomposition for the $G$-modules corresponding to $\pi$ and $\sigma$. See [Litt 1988] and [Koike 1987] for generalizations to the classical Lie groups (types $B_{n}, C_{n}, D_{n}$ ).

(iii) Since the work [Dema 1974] (cf. [Jos 1985]), an algebro-geometric treatment of the representation theory has led to many new results. We mention one. Suppose $\lambda, \mu \in$ $\Lambda^{+}(G, T)$. Recall from 5.1(vi) that there are $J, K \subseteq R$ such that the stabilizer $W_{\lambda}$ of $\lambda$ in $W$ coincides with $W_{J}$, and similarly $W_{\mu}=W_{K}$ for some $K \subseteq R$. Consider the map $\eta: D_{J, K} \rightarrow \Lambda^{+}(G, T)$ sending $w$ to $\overline{\lambda+w \mu}$ (cf. 5.1 and 9.9 for notation). Then, by [Kumar 1989], the following lower bound exists:

$$
(V(\lambda) \otimes V(\mu), V(\overline{\lambda+w \mu})) \geq\left|\eta^{-1}(\overline{\lambda+w \mu})\right|
$$

\section{The Hecke algebra}

The Hecke algebra is of use in the representation theory of finite groups of Lie type, for instance by decomposing the representation of such a group induced from the trivial one of a Borel subgroup into irreducibles. It also arose in the study of finite buildings. But a renewed interest in Hecke algebras came about as a consequence of the work of Kazhdan \& Lusztig [KaLu 1979]. Here, we merely introduce the basic notions and point out some relevant literature. [Curtis 1987] provides another brief overview. More elaborate treatments can be found in [Cart 1985], [CIK 1972], [Lusz 1984], and [MaSp 1988].

Choose a formal parameter $t$ and set $q=t^{2}$. The ring of coefficients we shall work with is $A=\mathbb{Z}\left[t, t^{-1}\right]$. The Hecke algebra is the free $A$-module with basis $\left\{a_{w}\right\}_{w \in W}$ supplied with the multiplication determined by

$$
a_{r} a_{w}=\left\{\begin{array}{ll}
a_{r w} & \text { if } l(r w)=l(w)+1 \\
(q-1) a_{w}+q a_{r w} & \text { if } l(r w)=l(w)-1
\end{array} \quad(r \in R ; w \in W) .\right.
$$

Thus, $H$ has unit $a_{1}$ and zero-divisors: $\left(a_{r}+1\right)\left(a_{r}-q\right)=0$. But the $a_{w}$ are invertible: for $r \in R$ the element $a_{r}^{-1}=q^{-1} a_{r}+\left(q^{-1}-1\right)$ satisfies $a_{r}^{-1} a_{r}=a_{1}$, so for arbitrary $w \in W$ with reduced decomposition $w=u v$, the inverse is $a_{w}^{-1}=a_{v}^{-1} a_{u}^{-1}$. In order to write these inverses in terms of the standard basis, we define the elements $R_{x, y} \in A$ by

$$
a_{y^{-1}}^{-1}=\sum_{x} s g(x y) q^{-l(y)} R_{x, y} a_{x}
$$


The following recursive relations help to compute the inverses explicitly:

$$
R_{x, y}= \begin{cases}0 & \text { if } x \not y \\ 1 & \text { if } x=y \\ R_{r x, r y} & \text { if } r x<x \text { and } r y<y \\ R_{x r, y r} & \text { if } x r<x \text { and } y r<y \\ (q-1) R_{r x, y}+q R_{r x, r y} & \text { if } r x>x \text { and } r y<y .\end{cases}
$$

Observe that $R_{x, y}$ is a polynomial in $q$ of degree $l(y)-l(x)$.

The choice of $t=q^{1 / 2}$ as a parameter rather than $q$ is related to the isomorphism, established in [Lusz 1981], between $H \otimes \mathbf{Q}(t)$ and the group algebra of $W$ over $\mathbf{Q}(t)$ in the case where $W$ is a Weyl group with connected diagram $M$, a result that is not true (for $M=E_{8}$ ) over $\mathbf{Q}(q)$. An explicit presentation of this isomorphism in the case $M=I_{2}^{m}$ already leads to vast computations, see [Fak 1989].

For $\alpha \in \mathbb{C}$, denote by $\mathbb{C}_{\alpha}$ the $A$-algebra determined by the morphism $A \rightarrow \mathbb{C}$ that substitutes $t$ by $\alpha$; and similarly for $\mathbb{Z}$ in place of $\mathbb{C}$. Then $H \otimes_{A} \mathbb{C}_{\alpha}$ is a $\mathbb{C}$-algebra, and the ring $H \otimes_{A} \mathbb{Z}_{1}$ is isomorphic to the group ring of $W$ over $\mathbb{Z}$.

10.1 Theorem (Semisimplicity, cf. [GyUn 1989]) Suppose $W$ is finite with connected diagram. Let $\alpha \in \mathbb{C}$. Then the $\mathbb{C}$-algebra $H \otimes_{A} \mathbb{C}_{\alpha}$ is semi-simple if and only if $\sum_{w \in W} \alpha^{2 l(w)}=$ 0 .

10.2 Example Let $\mathcal{C}$ be a finite building of type $M$. Construct the vector space $V=$ $\bigoplus_{c \in \mathcal{C}} \operatorname{IR} c$ of dimension $|\mathcal{C}|$. For each type $r \in R$, the endomorphism $\alpha_{r}$ of $V$ determined by $\alpha_{r}(c)=\sum_{d \in c r^{*}, d \neq c} d$ for $c \in \mathcal{C}$ satisfies $\alpha_{r}^{2}=\left(q_{r}-1\right) a_{r}+q_{r} i d_{V}$, where $q_{r}+1$ is the size of an $r$-cell. Also, for $w \in W$, the expression $\alpha_{r_{1}} \cdots \alpha_{r_{t}}$ does not depend on the choice of the reduced expression $r_{1} \cdots r_{t}$ for $w$, so that we refer to it by $\alpha_{w}$. Hence if all panels have the same size $q_{r}$ (independent of $r \in R$ ), we have a representation in $V$ of $H \otimes_{A} \mathbb{Z}_{q_{r}}$. In [Lusz 1983] a treatment of the Hecke algebra in which the parameter $q_{r}$ may vary is given. In this guise, the Hecke algebra plays a rôle in Kilmoyer and Solomon's proof of the Feit-Higman Theorem (cf. [BCN 1989]).

10.3 $W$-graphs A tool in the construction of representations of the Hecke algebra is the so-called $W$-graph. This is a triple $(X, I, \mu)$ consisting of a set $X$, a map $I: X \rightarrow \mathcal{P}(R)$, and a function $\mu: X \times X \rightarrow \mathbb{Z}$, such that $X$ is the basis of a free $A$-module affording a representation of $H$ via

$$
a_{r} x= \begin{cases}-x & \text { if } r \in I(x) \\ q x+t \sum_{y \in X, r \in I(y)} \mu(y, x) y & \text { otherwise }\end{cases}
$$

The specification $t \mapsto 1$ turns the representation involved into a Coxeter group representation. In [Gyoja 1984] it is shown that any complex representation of a Weyl group with connected diagram can be constructed by means of a $W$-graph. In [Heck 1988] (technically involved) necessary and sufficient conditions on $(W, I, \mu)$ are given for the triple to be a $W$-graph. In order to find smaller modules from the representations defined by a $W$-graph $\Gamma=(X, I, \mu)$, a preorder $\leq_{\Gamma}$ is introduced on $X$ in the following way: $x \leq_{\Gamma} y$ if there is a sequence $x=x_{0}, \ldots, x_{t}=y$ with $\mu\left(x_{i}, x_{i+1}\right) \neq 0$ and $I\left(x_{i}\right) \notin I\left(x_{i+1}\right)$ for each $i \in\{0, \ldots, t-1\}$. Observe that $x \leq_{\Gamma} y$ implies that $x$ occurs in the $H$-submodule of $\bigoplus_{z \in X} A z$ generated by $y$. We let $\sim_{\Gamma}$ stand for the equivalence relation given by $x \sim_{\Gamma} y$ if 
and only if $x \leq_{\Gamma} y \leq_{\Gamma} x$, and call the equivalence classes $\Gamma$-cells. Each $\Gamma$-cell $Y$ gives rise to a. $W$-graph, by restriction of $I$ and $\mu$.

The ring automorphism $a \mapsto \bar{a}(a \in A)$ determined by $\bar{t}=t^{-1}$ is an involution, which can be extended to an involution on $H$, also written $x \mapsto \bar{x}$, by requiring $\overline{a_{w}}=a_{w^{-1}}^{-1}$. In [KaLu 1979], the existence of ${ }^{-}$-fixed basis is established through the following result.

10.4 Theorem (Kazhdan-Lusztig polynomials) For any $w \in W$ there is a unique element $c_{w} \in H$ such that

$$
\begin{gathered}
\overline{c_{w}}=c_{w} \\
c_{w}=\sum_{y \leq w} s g(y w) t^{l(w)-2 l(y)} \overline{P_{y, w}} a_{y}
\end{gathered}
$$

where $P_{y, w} \in A$ is a polynomial in $q$ of degree at most $(l(w)-l(y)-1) / 2$ for $y<w$ and $P_{w, w}=1$.

As for the proof, the uniqueness of the $P_{x, y}$ follows from consideration of the identity

$$
P_{x, y}=\sum_{w \in W} q^{l(x)-l(y)} \overline{R_{x, w} P_{w, y}}
$$

which can be derived from equation (1a) by substituting (1b) at both sides and use of the polynomials $R_{x, y}$. Suppose uniqueness of the $P_{w, y}$ has been settled for all $w \in W$ with $x<w \leq y$; then the above identity yields

$$
P_{x, y}-q^{l(x)-l(y)} \overline{P_{x, y}}=\sum_{x<w} q^{l(x)-l(y)} \overline{R_{x, w} P_{w, y}}
$$

and the degree arguments show that $P_{x, y}$ is uniquely determined.

As for existence, first consider the following relation regarding elements $x, w \in W$ :

$x \prec w$ if $x<w, l(w)-l(x)$ is odd, and $P_{x, w}$ has degree $l(w)-l(x)-1$ in $t$

For $x \prec w$ write $\mu(x, w)$ for the coefficient of $t^{l(w)-l(x)-1}$ in $P_{x, w}$. Thus $\mu(x, w)$ is a nonzero integer.

Next, note that $c_{e}=a_{e}=1$. We proceed with the construction of $c_{w}$ and the $P_{y, w}$ for $y \leq w$ by induction on $l(w)$. Thus, let $w \in W$, and suppose $P_{z, w}$ and $z \prec w$ are well defined by the above rules for $z \leq w$. Then for $r \in R$ with $r w>w$, the relation

$$
c_{r w}=\left(t^{-1} a_{r}-t\right) c_{w}-\sum_{r z<z \prec w} \mu(z, w) c_{z},
$$

defines $c_{r w}$ and can be shown to satisfy (1a) and (1b) by use of the induction hypothesis. (For instance, (1a) comes down to $\overline{t^{-1} a_{r}-t}=t^{-1} a_{r}-t$, which is a direct consequence of the multiplication rule $a_{r}^{2}=(q-1) a_{r}+q$.)

For $w \in W$, put $\mathcal{L}(w)=\{r \in R \mid r w<w\}$. Here are two more relations regarding elements $x, w \in W$ :

$x \leq_{L} w$ if there is a sequence $x=x_{0}, \ldots, x_{t}=w$ with $x_{i}-x_{i+1}$ and $\mathcal{L}\left(x_{i}\right) \nsubseteq \mathcal{L}\left(x_{i+1}\right)$ for each $i \in\{0, \ldots, t-1\}$;

$x \leq_{L R} w$ if there is a sequence $x=x_{0}, x_{1} \ldots, x_{t}=w$ with $x_{i} \leq_{L} x_{i+1}$ and $x_{i}^{-1} \leq_{L} x_{i+1}^{-1}$ for each $i \in\{0, \ldots, t-1\}$. For $r \in R$ and $w \in W$, the following relations hold

$$
a_{r} c_{w}= \begin{cases}-c_{w} & \text { if } r w<w \\ q c_{w}+t c_{r w}+t \sum_{r x<x \prec w} \mu(x, w) c_{x} & \text { if } r w>w\end{cases}
$$


Therefore, the triple $\Gamma=(W, \mathcal{L}, \mu)$ is a $W$-graph. The corresponding preorder $\leq_{\Gamma}$ coincides with $\leq_{L}$. Its cells are called left cells.

By considering the opposite group $W^{0}$ (again a Coxeter group of type $M$ ) of $W$ acting on the right, the set $W$ can actually be turned into a $W \times W^{0}$-graph. Its preorder is $\leq_{L R}$, its cells are called two sided cells.

10.5 Examples (i) $M=A_{n}$. The Robinson-Schensted correspondence is a bijective map (effective!) between the set of all pairs of Standard Young Tableaux of size $n+1$ having the same shape and $W$. Via this correspondence, a two-sided cell consists of all permutations in $\operatorname{Sym}_{n+1}$ having the the same shape of Young Tableau, and a left cell consists of all permutations in $\mathrm{Sym}_{n+1}$ having the same first Young Tableau. See [Kerov 1985] for the symmetric groups on at most 6 letters, and [LaSch 1982] for a combinatorial approach to the Kazhdan-Lusztig polynomials.

(ii) Affine Weyl groups have a finite number of left (and right) cells (cf. [Lusz 1987]). This is not true for arbitrary Coxeter groups, not even if $|R|=3$, see [Béd 1989]. A number of small rank cases have been dealt with explicitly. See [Lawt 1989], [Shi 1986, 1989] for a cell decomposition in case of type $\tilde{A}$, using a generalization of Robinson-Schensted. Furthermore, [Béd 1986] for $\tilde{C}_{2}$, [Du 1988-90] for several other low rank types.

10.6 Explicit results The polynomials $P_{y, w}$ are hard to compute. The search for directer ways of determining the cells has received much attention. See [GaLa 1988] for a discussion. In [Alvis 1987] the results of a computation of left cells for $W\left(H_{4}\right)$ are given.

10.7 Remarks The Kazhdan-Lusztig conjectures (brought forward in [KaLu 1979]) describe how the $P_{y, w}(1)$ can be interpreted in terms of multiplicities in Verma modules; they have been established by [BrKa 1981] and [BeBe 1981]. See [BaVo 1982] and [KaLu 1987] for different applications.

Analogously to the way the Hecke algebra of a group of Lie type is related to the variety $G / B$, there is a version for $G / P$ where $P$ is a (so-called parabolic) subgroup of $G$ containing $B$. See [Deo 1987a] for a development of Kazhdan-Lusztig polynomials in this context.

\section{References}

[Alvis 1987] D. Alvis, The left cells of the Coxeter group of type $H_{4}, \mathrm{~J}$. Algebra, 107(1987), 160-168.

[Appel 1984] K. Appel, On Artin groups and Coxeter groups of large type, Contemp. Math., 33(1984), 50-78.

[ApSc 1983] K. Appel \& P.E. Schupp, Artin groups and infinite Coxeter groups, Inventiones Math., 72(1983), 201-220.

[BaVo 1982] D. Barbasch \& D. Vogan, Primitive ideals and orbital integrals in complex classical groups, Math. Ann., 259(1982), 153-199.

[BeBe 1981] A.A. Beilinson \& J. Bernstein, Localisation des g-modules, C.R. Acad. Sci. Paris, Serie I, 292(1981), 15-18.

[Béd 1986] R. Bédard, Cells for two Coxeter groups, Comm. Algebra, 14(1986), 12531286.

[Béd 1989] R. Bédard, Left V-Cells for hyperbolic Coxeter groups, Comm. Algebra, 17(1989), 2971-2997. 
[Bezv 1986] V.N. Bezverkhnii, Solution to the conjugacy problem for words in Artin and Coxeter groups of large type, Tulsk. Gos. Ped. Inst., Tula, 126(1986), 26-61.

[Bjö 1984] A. Björner, Orderings of Coxeter groups, Contemp. Math., AMS, 34(1984), 175-195.

[BjWa 1982] A. Björner \& M. Wachs, Bruhat order of Coxeter groups and shellability, Adv. in Math., 43(1982), 87-100.

[Bourb 1968] N. Bourbaki, Groupes et algèbres de Lie, Chap 4, 5, et 6, Hermann, Paris, 1968.

[BCN 1989] A.E. Brouwer, A.M. Cohen, A. Neumaier, Distance-regular Graphs, Ergebnisse der Math. u.i. Grenzgeb. 3. Folge Band 18, Springer-Verlag, Berlin, 1989.

[Brown 1989] K.S. Brown, Buildings, Springer-Verlag, New York, 1989.

[BrTi 1984] F. Bruhat \& J. Tits, Groupes réductifs sur un corps local, II, Publ. Math. I.H.E.S., 60(1984), 5-184.

[BrKa 1981] J.-L. Brylinski \& M. Kashiwara, Kazhdan-Lusztig conjecture and holonomic systems, Inventiones Math., 64(1981), 387-410.

[BuCo 1990] F. Buekenhout \& A.M. Cohen, Diagram Geometry, in preparation, 1990.

[Cart 1985] R.W. Carter, Finite groups of Lie type: conjugacy classes and complex characters, Wiley, Chichester, 1985.

[CoRu 1990] A.M. Cohen \& G.C.M. Ruitenburg, Generating functions and Lie Groups, preprint, (1990), 8pp.

[CNS 1988] J.H. Conway, S.P. Norton, L.H. Soicher, The Bimonster, the group $Y_{555}$ and the projective plane of order 3, pp. 27-50 in: Computers in Algebra (eds.: M.C. Tangora), Lecture Notes in Pure \& Applied Math. vol 111, Marcel Dekker, New York, 1988.

[CSW 1989] J.H. Conway, N.J.A. Sloane, A.R. Wilks, Gray codes for reflection groups, Graphs and Combinatorics, 5(1989), 315-325.

[Cox 1934] H.S.M. Coxeter, Finite groups generated by reflections and their subgroups generated by reflections, Proc. Cambridge Phil. Soc., 30(1934), 466-282.

[Curtis 1987] C.W. Curtis, The Hecke algebra of a finite group, Proc. of Symp. in Pure Math., 47(1987), 51-60.

[CIK 1972] C.W. Curtis, N. Iwahori, R. Kilmoyer, Hecke algebras and characters of parabolic type of finite groups with BN-pairs, Publ. Math. IHES, 40(1972), 81-116.

[delaH 1987] O. de la Harpe, Groupes de Coxeter infinis non-affines, Exposition. Math., 5(1987), 91-96.

[Deo 1977] V.V. Deodhar, Some characterizations of Bruhat ordering on a Coxeter group and determination of the relative Möbius Function, Inv. Math., 39(1977), 187-198.

[Deo 1982] V.V. Deodhar, On the root system of a Coxeter group, Comm. Algebra, 10(1982), 611-630.

[Deo 1986] V.V. Deodhar, Some characterizations of Coxeter groups, Enseignements Math., 32(1986), 111-120. 
[Deo 1987a] V.V. Deodhar, On some geometric aspects of Bruhat orderings II. The parabolic analogue of Kazhdan-Lusztig polynomials, J. Algebra, 111(1987a), 483-506.

[Deo 1987b] V.V. Deodhar, A splitting criterion for the Bruhat orderings on Coxeter groups, Comm. Algebra, 15(1987b), 1889-1894.

[Deo 1989] V.V. Deodhar, $A$ note on subgroups generated by reflections in Coxeter groups, Archiv d. Math., 53(1989), 543-546.

[DrSc 1988] A.W.M. Dress \& E. Schulte, $A$ local characterization of regular polytopes in terms of equivelarity, preprint, (1988), 10pp.

[Du 1988a] Du Jie, The decomposition into cells of the affine Weyl group of type $\tilde{B}_{3}$, Comm. Algebra, 16(1988a), 1383-1409.

[Du 1988b] Du Jie, Two-sided cells in the affine Weyl group of type $\tilde{C}_{3}$, J. London Math. Soc., 38(1988b), 87-98.

[Du 1990] Du Jie, Cells in the affine Weyl group of type $\tilde{D}_{4}$, J. Algebra, 128(1990), 384-404.

[Dyer 1990] M. Dyer, Reflection subgroups of Coxeter systems, preprint M.I.T., (1990), 14 pp.

[Fak 1989] A.P. Fakiolas, The Lusztig isomorphism for Hecke algebras of dihedral type, J. Algebra, 126(1989), 466-492.

[GaLa 1988] A.M. Garsia \& T.J. McLarnan, Relations between Young's natural and the Kazhdan-Lusztig representations of $\mathrm{Sym}_{n}$, Adv. Math., 69(1988), 32-92.

[Gyoja 1984] A. Gyoja, On the existence of a $W$-graph for an irreducible representation of a Coxeter group, J. Algebra, 86(1984), 422-438.

[GyUn 1989] A. Gyoja \& K. Uno, On the semisimplicity of Hecke algebras, J. Math. Soc. Japan, 41(1989), 75-79.

[Heck 1988] A. Heck, $A$ criterion for a triple $(X, I, \mu)$ to be a $W$-graph of a Coxeter group, Comm. Algebra, 16(1988), 2083-2102.

[Heckm 1982] G. Heckman, Projections of Orbits and Asymptotic Behavior of Multiplicities for Compact Connected Lie Groups, Inv. Math., 67(1982), 333-356.

[Hend 1985] H. Hendriks, Hyperplane complements of large type, Inv. Math., 79(1985), 375-381.

[Hill 1982] H. Hiller, Geometry of Coxeter Groups, Pitman, Boston, 1982.

[vdH 1974] A. van den Hombergh, About the automorphisms of the Bruhat-ordering in a Coxeter group, Indag. Math., 36(1974), 125-131.

[Howl 1988] R.B. Howlett, On the Schur multipliers of Coxeter groups, J. London Math. Soc., 38(1988), 263-276.

[Hum 1974] J.E. Humphreys, Introduction to Lie algebras and representation theory, Springer, New York, 1974.

[JaKe 1981] G. James, A. Kerber, The Representation Theory of the Symmetric Group, Encyclopedia of Mathematics and its Applications, 16, Addison-Wesley, New York, 1981.

[Jantz 1987] J.C. Jantzen, Representations of Algebraic Groups, Acad. Press, New York, 1987. 
[Jos 1985] A. Joseph, On the Demazure character formula, Ann. Sc. Éc. Norm. Sup., 18(1985), 389-419.

[Kac 1985] V.G. Kac, Infinite dimensional Lie algebras, 2nd ed., Cambridge University Press, Cambridge, 1985.

[KaLu 1979] D. Kazhdan \& G. Lusztig, Representations of Coxeter Groups and Hecke algebras, Inv. Math., 53(1979), 165-184.

[KaLu 1987] D. Kazhdan \& G. Lusztig, Proof of the Deligne-Langlands conjecture for Hecke algebras, Inv. Math., 87(1987), 153-215.

[Kane 1987] M. Kaneda, On the inverse Kazhdan-Lusztig polynomials for affine Weyl groups, J. reine angew. Math., 381(1987), 116-135.

[Kerov 1985] S. V. Kerov, W-graphs of representations of symmetric groups, J. Sov. Math., 28(1985), 596-605.

[King 1971] R.C. King, Branching rules for classical Lie groups using tensor and spinor methods, J. Phys., A8(1971), 429-449.

[Koike 1987] K.Koike, On new multiplicity-formulas of weights of representations for the classical groups, J. Algebra, 107(1987), 512-533.

[Koike 1987] K. Koike, I. Terada, Young-diagrammatic methods for the representation theory of the classical groups of type $B_{n}, C_{n}, D_{n}$, J. Algebra, 107(1987), 466-511.

[Kumar 1988] S. Kumar, Proof of the Pathasarathy-Ranga Rao-Varadarajan conjecture, Inv. Math., 93(1988), 117-130.

[Kumar 1989] S. Kumar, A refinement of the PRV-conjecture, Inv. Math., 97(1989), 305311.

[LakSe 1986] V. Lakshmibai \& C.S. Seshadri, Geometry of G/P - V, J. Algebra, 100(1986), 462-557.

[LaSch 1982] A. Lascoux \& M.-P. Schützenberger, Polynomes de Kazhdan-Lusztig pour les Grassmanniennes, Astérisque, 87-88(1982), 249-266.

[Lawt 1989] G. Lawton, Two-sided cells in the affine Weyl group of type $\tilde{A}_{n-1}$, J. Algebra, 120(1989), 74-89.

[LeCh 1986] P. Le-Chenadec, Canonical forms in finitely presented algebras, Pitman, London, 1986.

[Litt 1987] P. Littelmann, A decomposition formula for Levi subgroups, preprint, (1987), 1-14.

[Litt 1988] P. Littelmann, A Littlewood-Richardson rule for classical groups, C.R. Acad. Sci., Tome 306, série, 1, no 6(1988), 299-303.

[Lusz 1981] G. Lusztig, On a theorem of Benson and Curtis, J. Algebra, 71(1981), 490498.

[Lusz 1983] G. Lusztig, Left cells in Weyl groups, Lie group representations, I SpringerVerlag Lecture Notes in Math., 1024(1983), 99-111.

[Lusz 1984] G. Lusztig, Characters of reductive groups over a finite field, Annals of Math. Studies 107, Princeton Univ. Press, 1984.

[Lusz 1985] G. Lusztig, Cells in affine Weyl groups, Algebraic groups and related topics, Advanced Studies in Pure Math., 6(1985), 255-287.

[Lusz 1987] G. Lusztig, Cells in affine Weyl groups, II, J. Algebra, 109(1987), 536-548. 
[Macd 1979] I.G. Macdonald, Symmetric functions and Hall polynomials, Oxford University Press, Oxford, 1979.

[MaSp 1988] J.G.M. Mars \& T.A. Springer, Character sheaves, preprint RUU, 552(1988), 88pp.

[Mat 1988] O. Mathieu, Formule de charactère pour les algèbres de Kac-Moody générales, Astérisque, (1988), 159-160.

[McPa 1981] W.G. McKay, J. Patera, Tables of dimensions, indices, and branching rules for representations of simple Lie algebras, Marcel Dekker, New York, 1981.

[McMu 1967] P. McMullen, Combinatorially regular polytopes, Mathematika, 14(1967), 142-150.

[Neid 1986] W. Neidhardt, An algorithm for computing weight multiplicities in irreducible highest weight modules over Kac-Moody algebras, Algebras, Groups, Geom., 3(1986), 19-26.

[RiSp 1989] R.W. Richardson \& T.A. Spinger, The Bruhat order on symmetric varieties, RUU preprint, 597(1989), 66pp.

[Ron 1989] M. Ronan, Lecture on Buildings, Acad. Press, Boston, 1989.

[Ser 1987] J.-P. Serre, Complex Semisimple Lie algebras, Springer Verlag, Berlin, 1987.

[Shi 1986] Jian-Yi Shi, The Kazhdan-Lusztig cells in certain affine Weyl groups, Lecture Notes in Math. 1179, Springer-Verlag, Berlin, 1986.

[Shi 1987] Shi Jian-yi, Alcoves corresponding to an affine Weyl group, J. London Math. Soc., 35(1987), 42-55.

[Shi 1987] Shi Jian-yi, Sign types corresponding to an affine Weyl group, J. London Math. Soc., 35(1987), 56-74.

[Shi 1987] Shi Jian-yi, $A$ two-sided cell in an affine Weyl group, J. London Math. Soc., 36(1987), 407-420.

[Shi 1988] Shi Jian-yi, A two-sided cell in an affine Weyl group, II, J. London Math. Soc., 37(1988), 253-264.

[Shi 1989] Shi Jian-yi, Some recent developments on the cells of affine Weyl groups, Contemporary Math., 82(1989), 159-169.

[Spri 1981] T.A. Springer, Linear algebraic groups, Birkhäuser, Basel, 1981.

[Spri 1982] T.A. Springer, Some remarks on involutions in Coxeter groups, Comm. Algebra, 10(1982), 631-636.

[Stan 1984] R. Stanley, On the number of reduced decompositions of elements of Coxeter groups, European J. Combinatorics, 5(1984), 359-372.

[Tits 1969] J. Tits, Le problème des mots dans les groupes de Coxeter, Sympos. Math. Rome 1967/1968, Acad. Press, London, 1(1969), 175-185.

[Tits 1974] J. Tits, Buildings of spherical type and finite $B N$-pairs, Lecture Notes in Math. 386, Springer-Verlag, 1974.

[Tits 1981] J. Tits, A local approach to buildings, pp. 317-322 in: The Geometric Vein (Coxeter Festschrift) (eds.: Ch. Davies, B. Grünbaum, F.A. Scherk), Springer-Verlag, 1981.

[Tits 1988] J. Tits, Sur le groupe des automorphisms de certains groupes de Coxeter, J. Algebra, 113(1988), 346-357. 
[Tsar 1990] S.V. Tsaranov, Representation and classification of Coxeter monoids, preprint, Moscow, (1990), 24pp.

[Var 1984] V.S. Varadarajan, Lie Groups, Lie Algebras, and their Representations, Graduate Texts in Math. 102, Springer, New York, 1984.

[Vinb 1971] E. Vinberg, Discrete linear groups generated by reflections, Math USSR Izvestija, 5(1971), 1083-1119.

[Vret 1988] L. Vretare, Character calculations for semisimple Lie algebras, Lund Institute of Technology Report, 11(1988), 14 pp.

[Wag 1980/1] A. Wagner, Determination of the finite primitive reflection groups over an arbitrary field of characteristic not 2, I,II,III, Geom. Dedicata, 9,10,10(1980/1), 239-253, 191-203, 475-523.

[Wate 1989] W.C. Waterhouse, Automorphisms of the Bruhat order on Coxeter groups, Bull. London Math. Soc., 21(1989), 243-248.

[ZaSe 1981] A.E. Zalesskii \& V.N. Serezkin, Finite linear groups generated by reflections, Math. USSR Izvestija, 17(1981), 477-503.

[Zales 1983] A.E. Zalesskii, The fixed algebra of a group generated by reflections is not always free, Arch. Math., 41(1983), 434-437.

Added in proof. A few months after this manuscript had been finished I discovered that a new book on Coxeter groups had come into print: "Reflection groups and Coxeter groups" by J.E. Humphreys (Cambridge University Press, 1990). It gives a transparent and extensive account of the basic theory and some of the topics dealt with in this paper (such as Hecke algebras). 